\title{
RELAXATION OF A CONTROL PROBLEM IN THE COEFFICIENTS WITH A FUNCTIONAL OF QUADRATIC GROWTH IN THE GRADIENT*
}

\author{
JUAN CASADO-DÍAZ ${ }^{\dagger}$, JULIO COUCE-CALVO ${ }^{\dagger}$, AND JOSÉ D. MARTÍN-GÓMEZ†
}

\begin{abstract}
We study an optimal design problem consisting in mixing two anisotropic (electric or thermal) materials in order to minimize a functional depending on the gradient of the state. It is known that this type of problem has no solution in general, and then it is necessary to introduce a relaxed formulation. Here we prove that this relaxation is obtained by using composite materials, is constructed by homogenization, and takes a particular extension of the cost functional to these new materials. We obtain an integral representation of this relaxed cost functional. Besides, we show that our results contain some previous results obtained by other authors for isotropic materials.
\end{abstract}

Key words. control in the coefficients, elliptic PDE, optimal design

AMS subject classification. 49K20

DOI. $10.1137 / 070685890$

1. Introduction. We consider a control problem for a linear elliptic partial differential equation where the control variable is the diffusion matrix (control problem in the coefficients). This type of problem appears in optimal design. Recall that the thermic or electric properties of a material are given by the corresponding diffusion matrix, and so choosing an optimal matrix diffusion is equivalent to choosing an optimal material. To simplify the exposition we consider a two-phase optimization problem; i.e., we assume that the materials are constructed by mixing two fixed materials (nonisotropic in general) represented by their diffusion matrices $A$ and $B$, which we take to be symmetric and elliptic.

As a model problem we consider the following one: For a bounded open set $\Omega \subset \mathbf{R}^{N}$, we look for a measurable set $\omega \subset \Omega$ such that for a given source term $f \in L^{2}(\Omega)$ (or more generally in $H^{-1}(\Omega)$ ) the solution $u$ of

$$
\left\{\begin{array}{l}
-\operatorname{div}\left(A \chi_{\omega}+B \chi_{\Omega \backslash \omega}\right) \nabla u=f \text { in } \Omega, \\
u=0 \text { on } \partial \Omega
\end{array}\right.
$$

minimizes a given functional $J$ on the Sobolev space $H_{0}^{1}(\Omega)$. We also assume that the measure of $\omega$ is less than or equal to $\kappa|\Omega|$, with $0<\kappa<1$; i.e., we dispose only of a limited quantity of material $A$.

It is well known (see, e.g., [20], [21]) that, in general, this problem has no solution (some existence results can be obtained in particular situations [12]), and so it is necessary to relax the problem. By denoting, for $p \in[0,1], \mathcal{K}(A, B, p)$ as the set of matrices constructed via homogenization, mixing $A$ and $B$ with respective proportions $p$ and $1-p$, and assuming $J$ sequentially continuous for the weak topology of $H_{0}^{1}(\Omega)$, it is well known (see, e.g., [1], [11], [16], [17], [22], [24], [26], [27], [29]) that the relaxation

\footnotetext{
${ }^{*}$ Received by the editors March 21, 2007; accepted for publication (in revised form) December 3, 2007; published electronically May 7, 2008. This work was supported by projects MTM2005-04914 of the Ministerio de Eduación y Ciencia of Spain and FQM309 of the Junta de Andalucía.

http://www.siam.org/journals/sicon/47-3/68589.html

${ }^{\dagger}$ Dpto. de Ecuaciones Diferenciales y Análisis Numérico, Facultad de Matemáticas, C. Tarfía s/n, 41012 Sevilla, Spain (jcasadod@us.es, couce@us.es, jdmartin@us.es).
} 
of the model control problem is obtained by replacing the set of controls

$$
\left\{A \chi_{\omega}+B \chi_{\Omega \backslash \omega}: \omega \subset \Omega \text { measurable, }|\omega| \leq \kappa|\Omega|\right\}
$$

by the larger set

$$
\left\{(M, \theta) \text { measurable : } \theta \in[0,1], M \in \mathcal{K}(A, B, \theta) \text { a.e. in } \Omega, \int_{\Omega} \theta d x \leq \kappa|\Omega|\right\} \text {. }
$$

In the present paper we are interested in functionals depending on the gradient of the state function, and so they are not sequentially continuous in general on the weak topology of $H_{0}^{1}(\Omega)$. For simplicity, we restrict ourselves to the functional

$$
J(u)=\int_{\Omega} F(\nabla u) d x+G(u), \quad u \in H_{0}^{1}(\Omega),
$$

where $F$ is a Hölder-continuous function in $\mathbf{R}^{N}$ with a quadratic growth and $G$ is sequentially continuous for the weak topology of $H_{0}^{1}(\Omega)$. Thus, the control problem considered in this paper is given by

$$
\left\{\begin{array}{l}
\inf \left\{\int_{\Omega} F(\nabla u) d x+G(u)\right\}, \\
-\operatorname{div}\left(A \chi_{\omega}+B \chi_{\Omega \backslash \omega}\right) \nabla u=f \text { in } \Omega, \\
u \in H_{0}^{1}(\Omega), \quad \omega \subset \Omega \text { measurable, }|\omega| \leq \kappa|\Omega| .
\end{array}\right.
$$

Some control problems related to (1.4) have also been considered by other authors. In this way, for the case

$$
A=\alpha I, \quad B=\beta I, \quad J(u)=\int_{\Omega}|\nabla(u-v)|^{2} d x
$$

it is proved in [30] that there exists a dense subset of $v \in H^{1}(\Omega)$ such that, by taking as control variable the functions of $L^{\infty}(\Omega)$ valuated in $[0,1]$, instead of the characteristic functions of measurable subsets of $\Omega$, problem (1.4) has a unique solution (the fact that $A=\alpha I, B=\beta I$ is not relevant in the reasoning used in [30]).

Related to this result, we mention that, for $N=2, A=\alpha I, B=\beta I$, and $F(\xi)=G(|\xi|)$, convex in $\xi$, and growth not necessarily quadratic, it is proved in [25] that a relaxation of (1.4) can be obtained by just replacing the characteristic functions by functions valuated in $[0,1]$.

On the other hand, a relaxation of problem (1.4) when $A, B$, and $J$ are given by (1.5) is obtained in [3], [10], [18]. We also refer to [15], [31], where, from a partial relaxation, it is realized a numerical study of the problem.

Some relaxation and numerical results for (1.4) have also been obtained in [2] when $A$ and $B$ are not necessarily scalar matrices but $B-A$ is small.

Other relaxation problems for anisotropic materials (in diffusion and elasticity) have been considered in [12], [13], [14], where the functional is sequentially continuous with respect to the weak topology of $H_{0}^{1}(\Omega)$, but constraints appear on the gradients of the state functions.

In the present paper, for general $A, B$, and $F$, we show that the relaxation of (1.4) is given by replacing (as when $J$ was sequentially continuous in the weak topology of $H_{0}^{1}(\Omega)$ ) the set of controls (1.1) by (1.2) and the functional $J$ by

$$
\int_{\Omega} H(\nabla u, M \nabla u, \theta) d x+G(u)
$$

Copyright (C) by SIAM. Unauthorized reproduction of this article is prohibited. 
where $H:\left\{(\xi, \eta, p) \in \mathbf{R}^{N} \times \mathbf{R}^{N} \times[0,1]: \eta \in \mathcal{K}(A, B, p) \xi\right\} \rightarrow \mathbf{R}$ is defined by (4.6).

We prove that the function $H$ is continuous, satisfies the growth condition

$$
|H(\xi, \eta, p)| \leq C\left(1+|\xi|^{2}\right),
$$

and has some convexity properties given in Proposition 4.6.

We also obtain a characterization of the set $\mathcal{K}(A, B, p) \xi$ for every $\xi \in \mathbf{R}^{N}$, and we explicitly calculate $H$ on

$$
\left\{(\xi, \eta, p) \in \mathbf{R}^{N} \times \mathbf{R}^{N} \times[0,1]: \eta \in \partial \mathcal{K}(A, B, p) \xi\right\}
$$

(here $\partial$ denotes the boundary with respect to the affine hull). This can be useful to the study of optimality conditions for the relaxed problem. We refer to [1], [5], [6], [16], [24] for the study of optimality conditions for control problems in the coefficients.

Finally, we obtain an explicit expression of $H$ in its whole domain for the case where

$$
F(\xi)=s A \xi \cdot \xi
$$

where $s \in \mathbf{R}$ and $s(A-B)$ is nonnegative. This example contains in particular (see Corollary 6.2) the case where $A, B$, and $J$ are given by (1.5).

Although, to simplify the exposition, we have assumed that $J$ is given by (1.3), our techniques apply for a more general functional given by

$$
\int_{\omega} F_{1}(x, u, \nabla u) d x+\int_{\Omega \backslash \omega} F_{2}(x, u, \nabla u) d x+G(u),
$$

with $G$ as above and $F_{1}, F_{2}$ satisfying similar conditions to $F$. It is also possible to consider more than two materials (but then, we do not have an explicit characterization of the domain of $H$ ) and the more realistic case where the set of materials is invariable by rotations.

2. Notation. The space of linear applications from $\mathbf{R}^{N}$ into $\mathbf{R}^{N}$, which we assume to be identified with the space of matrices of dimension $N \times N$, is denoted by $\mathcal{M}_{N}$. The subspace of $\mathcal{M}_{N}$ corresponding to the symmetric matrices is denoted by $\mathcal{M}_{N}^{s}$.

The kernel and the range of $M \in \mathcal{M}_{N}$ are, respectively, denoted by $\operatorname{Ker}(M)$ and $\operatorname{Ran}(M)$.

For $M \in \mathcal{M}_{N}^{s}$, not necessarily invertible, we define $M^{\dagger}: \operatorname{Ran}(M) \rightarrow \operatorname{Ran}(M)$ the pseudoinverse of $M$, i.e., the inverse of the restriction of $M$ to its range.

The unitary cube in $\mathbf{R}^{N},(0,1)^{N}$ is denoted by $Y$.

For two sets $Z$ and $Z^{\prime}$, we denote by $Z \triangle Z^{\prime}$ its symmetric difference; i.e., $Z \triangle Z^{\prime}=$ $\left(Z \backslash Z^{\prime}\right) \cup\left(Z^{\prime} \backslash Z\right)$.

We use the subindex $\sharp$ to mean $Y$-periodicity. For example, the space of functions in the Sobolev space $H_{l o c}^{1}\left(\mathbf{R}^{N}\right)$ which are $Y$-periodic is denoted by $H_{\sharp}^{1}(Y)$. Indeed, in the present paper all of the functions defined on $Y$ are assumed to be extended to $\mathbf{R}^{N}$ by $Y$-periodicity.

Throughout the paper we denote by $A$ and $B$ two fixed positive symmetric matrices.

We define $\Xi: \operatorname{Ran}(A-B) \rightarrow A^{-1} \operatorname{Ran}(A-B)\left(=B^{-1} \operatorname{Ran}(A-B)\right)$ by

$$
\Xi \zeta=\nu \Leftrightarrow\left\{\begin{array} { l } 
{ ( B - A ) \nu = \zeta } \\
{ A \nu \in \operatorname { R a n } ( A - B ) }
\end{array} \Leftrightarrow \left\{\begin{array}{l}
(B-A) \nu=\zeta \\
B \nu \in \operatorname{Ran}(A-B)
\end{array}\right.\right.
$$

Copyright ( $)$ by SIAM. Unauthorized reproduction of this article is prohibited. 
for every $\zeta \in \operatorname{Ran}(A-B)$ or, equivalently,

$$
\Xi=(r A+s B)^{-1}(B-A)\left((B-A)(r A+s B)^{-1}(B-A)\right)^{\dagger}
$$

for every $r, s \geq 0, r+s>0$. Note that if $B-A$ is invertible, then $\Xi=(B-A)^{-1}$.

For $p \in[0,1]$, we define $\Lambda_{p}$ as the arithmetic mean of $A$ and $B$ with respective proportions $p$ and $1-p$; i.e.,

$$
\Lambda_{p}=p A+(1-p) B
$$

We denote by $\Omega \subset \mathbf{R}^{N}$ a fixed bounded open set, smooth enough for Meyer's theorem [19] to be satisfied and such that there exist $\hat{\Omega}$ open bounded with $\bar{\Omega} \subset \hat{\Omega}$ and a linear continuous prolongation operator from $H^{1}(\Omega)$ into $H_{0}^{1}(\hat{\Omega})$.

For a sequence $M_{n} \in L^{\infty}\left(\Omega ; \mathcal{M}_{N}\right)$, uniformly elliptic and bounded, and $M \in$ $L^{\infty}\left(\Omega ; \mathcal{M}_{N}\right)$, we write $M_{n} \stackrel{H}{\rightarrow} M$ to mean that $M_{n}$ converges to $M$ in the sense of the $H$-convergence [22], [27]. Indeed, as we usually deal with symmetric matrices, $H$-convergence is equivalent to the $G$-convergence of Spagnolo [26].

For $p \in[0,1]$ we denote by $\mathcal{K}(A, B, p)$ the set of materials constructed via homogenization mixing the materials corresponding to the diffusion matrices $A$ and $B$, with respective proportions $p$ and $1-p$; i.e.,

$$
\begin{aligned}
& \mathcal{K}(A, B, p)=\left\{M \in \mathcal{M}_{N}^{s}: \exists \omega_{n} \subset \mathbf{R}^{N}\right. \text { measurable, } \\
&\left.\chi_{\omega_{n}} \stackrel{*}{\rightarrow} p \text { in } L^{\infty}\left(\mathbf{R}^{N}\right), A \chi_{\omega_{n}}+B\left(1-\chi_{\omega_{n}}\right) \stackrel{H}{\rightarrow} M\right\} .
\end{aligned}
$$

Clearly, $\mathcal{K}(A, B, 1)=\{A\}, \mathcal{K}(A, B, 0)=\{B\}$.

For $\xi \in \mathbf{R}^{N}, p \in[0,1]$ we write

$$
\mathcal{K}(A, B, p) \xi=\left\{\eta \in \mathbf{R}^{N}: \exists M \in \mathcal{K}(A, B, p), \text { with } \eta=M \xi\right\} .
$$

3. Preliminary results. To our knowledge, an explicit characterization of the set $\mathcal{K}(A, B, p)$ is known only for isotropic materials (see, e.g., [17], [29]). Fortunately, for the purpose of the present paper, we need only to know the set $\mathcal{K}(A, B, p) \xi$ for every $\xi \in \mathbf{R}^{N}$. A characterization of this set is obtained in the present section.

We recall the following result due to Dal Maso and Kohn [9] (see also [1], [11]), which shows that the set $\mathcal{K}(A, B, p)$ can be obtained via periodic homogenization.

Theorem 3.1. For $p \in[0,1]$, the set of matrices $M$ for which there exists $Z \subset Y$, with $|Z|=p$, such that for every $\xi \in \mathbf{R}^{N}$,

$$
M \xi=\int_{Y}\left(A \chi_{Z}+B \chi_{Y \backslash Z}\right)(\xi+\nabla w) d y
$$

with $w$ the solution of

$$
\left\{\begin{array}{l}
w \in H_{\sharp}^{1}(Y), \quad \int_{Y} w d y=0, \\
-\operatorname{div}\left(\left(A \chi_{Z}+B \chi_{Y \backslash Z}\right)(\xi+\nabla w)\right)=0 \quad \text { in } \mathbf{R}^{N},
\end{array}\right.
$$

is dense in $\mathcal{K}(A, B, p)$.

The following result gives some properties of the solution $w$ of (3.2).

Copyright $@$ by SIAM. Unauthorized reproduction of this article is prohibited. 
Lemma 3.2. For $p \in(0,1)$, we consider $\xi, \eta \in \mathbf{R}^{N}$ and $Z \subset Y$ measurable, with $|Z|=p$, such that the solution $w$ of (3.2) satisfies

$$
\int_{Y}\left(A \chi_{Z}+B \chi_{Y \backslash Z}\right)(\xi+\nabla w) d y=\eta
$$

Then the following equalities hold:

$$
\begin{gathered}
\int_{Z} A \nabla w \cdot \nabla w d y+\int_{Y \backslash Z} B \nabla w \cdot \nabla w d y=\left(\Lambda_{p} \xi-\eta\right) \cdot \xi \\
(B-A) \int_{Z} \nabla w d y=-(B-A) \int_{Y \backslash Z} \nabla w d y=\Lambda_{p} \xi-\eta \\
\left\{\begin{array}{l}
\int_{Z} A\left(\nabla w-\frac{1}{p} \int_{Z} \nabla w d z\right) \cdot\left(\nabla w-\frac{1}{p} \int_{Z} \nabla w d z\right) d y \\
+\int_{Y \backslash Z} B\left(\nabla w-\frac{1}{1-p} \int_{Y \backslash Z} \nabla w d z\right) \cdot\left(\nabla w-\frac{1}{1-p} \int_{Y \backslash Z} \nabla w d z\right) d y \\
=\left(\Lambda_{p} \xi-\eta\right) \cdot \xi-\left(\frac{A}{p}+\frac{B}{1-p}\right)\left(\int_{Z} \nabla w d y\right) \cdot\left(\int_{Z} \nabla w d y\right) .
\end{array}\right.
\end{gathered}
$$

Proof. By using $w$ as a test function in (3.2), and taking into account (3.3), we easily get (3.4).

Since $w$ is periodic, we have

$$
\int_{Z} \nabla w d y+\int_{Y \backslash Z} \nabla w d y=0 .
$$

On the other hand, by (3.3) we obtain

$$
A \int_{Z} \nabla w d y+B \int_{Y \backslash Z} \nabla w d y=\eta-\Lambda_{p} \xi
$$

From these equalities we conclude (3.5).

To prove (3.6), it is enough to develop the left-hand side and then use (3.4).

As a consequence of Lemma 3.2, we have the following.

Proposition 3.3. If $N=1, p \in[0,1]$, we have

$$
\mathcal{K}(A, B, p)=\left\{\left(\frac{p}{A}+\frac{1-p}{B}\right)^{-1}\right\} .
$$

If $N \geq 2, p \in(0,1), \xi \in \mathbf{R}^{N}$, then, by denoting by $E(\xi, p)$ the ellipsoid

$$
E(\xi, p)=\left\{\nu \in \mathbf{R}^{N}:\left(\frac{A}{p}+\frac{B}{1-p}\right) \nu \cdot \nu \leq(B-A) \nu \cdot \xi\right\}
$$

we have

$$
\mathcal{K}(A, B, p) \xi=\Lambda_{p} \xi+(A-B) E(\xi, p) .
$$

Copyright $@$ by SIAM. Unauthorized reproduction of this article is prohibited. 
Proof. The case $N=1$ is well known. Indeed, it can be easily obtained by using the fact that for every $Z \subset Y$, with $|Z|=p$, the solution $w$ of (3.2) satisfies

$$
\xi+\frac{d w}{d x}= \begin{cases}\frac{\eta}{A} & \text { a.e. in } Z, \\ \frac{\eta}{B} & \text { a.e. in } Y \backslash Z,\end{cases}
$$

with

$$
\eta=\left(\frac{p}{A}+\frac{1-p}{B}\right)^{-1} \xi
$$

Assume now that $N \geq 2, p \in(0,1)$. For $Z \subset Y$, with $|Z|=p$, and the $w \in H_{\sharp}^{1}(Y)$ solution of (3.2), the left-hand side of (3.6) is nonnegative, and so equality (3.5) proves that the vector

$$
\nu=\int_{Z} \nabla w d y
$$

is in $E(\xi, p)$ and satisfies $\eta=\Lambda_{p} \xi+(A-B) \nu$. Since by Theorem 3.1 the set of $\eta$ constructed in this way is dense in $\mathcal{K}(A, B, p) \xi$, we then deduce the inclusion

$$
\mathcal{K}(A, B, p) \xi \subset \Lambda_{p} \xi+(A-B) E(\xi, p) .
$$

Reciprocally, let us now prove that every $\nu \in E(\xi, p)$ satisfies that $\Lambda_{p} \xi+(A-B) \nu$ belongs to $\mathcal{K}(A, B, p) \xi$.

If $\nu$ belongs to $\partial E(\xi, p) \backslash\{0\}$, this can be easily shown by using a lamination of $A$ and $B$ with respective proportions $p$ and $1-p$ in the direction of $\nu$. If $\nu=0$, we consider a lamination as above but now in an orthogonal direction to $(B-A) \xi$.

If $\nu$ belongs to the interior of $E(\xi, p)$, then, for $\lambda>1$ such that $\lambda \nu$ belongs to $\partial E(\xi, p)$, we take two matrices $M_{1}, M_{2} \in \mathcal{K}(A, B, p)$ such that $M_{1} \xi=\Lambda_{p} \xi, M_{2} \xi=$ $\Lambda_{p} \xi+\lambda(A-B) \nu$. A lamination of $M_{1}$ and $M_{2}$ with respective proportions $1-1 / \lambda$ and $1 / \lambda$ in an orthogonal direction to $\left(M_{2}-M_{1}\right) \xi$ provides a matrix $M \in \mathcal{K}(A, B, p)$ such that $\Lambda_{p} \xi+(A-B) \nu=M \xi$.

Corollary 3.4. For $N \geq 2, p \in(0,1)$, and $\xi \in \mathbf{R}^{N}$, we have

$$
\begin{aligned}
& \mathcal{K}(A, B, p) \xi=\left\{\eta \in \mathbf{R}^{N}: \Lambda_{p} \xi-\eta \in \operatorname{Ran}(A-B),\right. \\
& \left.\left((A-B)\left(\frac{A}{p}+\frac{B}{1-p}\right)^{-1}(A-B)\right)^{\dagger}\left(\Lambda_{p} \xi-\eta\right) \cdot\left(\Lambda_{p} \xi-\eta\right) \leq \xi \cdot\left(\Lambda_{p} \xi-\eta\right)\right\} .
\end{aligned}
$$

Proof. By Proposition 3.3, we have that $\eta$ belongs to $\mathcal{K}(A, B, p) \xi$ if and only if there exists $\nu \in \mathbf{R}^{N}$, such that $\Lambda_{p} \xi-\eta=(B-A) \nu$ and

$$
\left(\frac{A}{p}+\frac{B}{1-p}\right) \nu \cdot \nu \leq\left(\Lambda_{p} \xi-\eta\right) \cdot \xi
$$

or, equivalently, if and only if $\Lambda_{p} \xi-\eta \in \operatorname{Ran}(A-B)$ and

$$
\min \left\{\left(\frac{A}{p}+\frac{B}{1-p}\right) \nu \cdot \nu: \Lambda_{p} \xi-\eta=(B-A) \nu\right\} \leq\left(\Lambda_{p} \xi-\eta\right) \cdot \xi .
$$

Copyright $@$ by SIAM. Unauthorized reproduction of this article is prohibited. 
The minimum of this problem is attained in $\nu=\Xi\left(\Lambda_{p} \xi-\eta\right)$, with $\Xi$ defined by (2.1). By using the fact that by $(2.2)$

$$
\Xi=\left(\frac{A}{p}+\frac{B}{1-p}\right)^{-1}(B-A)\left((B-A)\left(\frac{A}{p}+\frac{B}{1-p}\right)^{-1}(B-A)\right)^{\dagger},
$$

we obtain (3.11).

As a consequence of this result, we have the following.

Corollary 3.5. For $N \geq 2, p \in(0,1), \xi \in \mathbf{R}^{N}$, the set $\mathcal{K}(A, B, p) \xi$ reduces to $\left\{\Lambda_{p} \xi\right\}$ if and only if $\xi \in \operatorname{Ker}(A-B)$.

Proof. By Corollary 3.4, we have $\mathcal{K}(A, B, p) \xi=\left\{\Lambda_{p} \xi\right\}$, if and only if for every $\zeta \in \operatorname{Ran}(A-B), \zeta \neq 0$, one has

$$
\left((A-B)\left(\frac{A}{p}+\frac{B}{1-p}\right)^{-1}(A-B)\right)^{\dagger} \zeta \cdot \zeta>\xi \cdot \zeta
$$

but this is equivalent to $\xi \in \operatorname{Ran}(A-B)^{\perp}=\operatorname{Ker}(A-B)$.

4. Formulation of the problem and main results. Let us consider a function $F: \mathbf{R}^{N} \rightarrow \mathbf{R}$ such that there exist $L>0, \varrho \in(0,1]$ which satisfy

$$
\left|F(\xi)-F\left(\xi^{\prime}\right)\right| \leq L\left(1+|\xi|+\left|\xi^{\prime}\right|\right)^{2-\varrho}\left|\xi-\xi^{\prime}\right|^{\varrho} \quad \forall \xi, \xi^{\prime} \in \mathbf{R}^{N} .
$$

Without loss of generality, we can also assume that

$$
F(0)=0
$$

These properties imply that $F$ satisfies

$$
|F(\xi)| \leq L(1+|\xi|)^{2} \quad \forall \xi \in \mathbf{R}^{N} .
$$

For the open set $\Omega$ and the matrices $A, B$ given in section 2, our aim here is to obtain a relaxation of the problem

$$
\left\{\begin{array}{l}
\inf \left\{\int_{\Omega} F(\nabla u) d x+G(u)\right\}, \\
-\operatorname{div}\left(A \chi_{\omega}+B \chi_{\Omega \backslash \omega}\right) \nabla u=f \text { in } \Omega, \\
u \in H_{0}^{1}(\Omega), \quad \omega \subset \Omega \text { measurable, }|\omega| \leq \kappa|\Omega|,
\end{array}\right.
$$

where $G$ is a sequentially continuous functional in the weak topology of $H_{0}^{1}(\Omega), f \in$ $H^{-1}(\Omega)$, and $\kappa \in(0,1)$. For this purpose, given $\delta>0$, we define $H_{\delta}: \mathbf{R}^{N} \times \mathbf{R}^{N} \times$ $[0,1] \rightarrow \mathbf{R} \cup\{+\infty\}$ by

$$
\left\{\begin{aligned}
H_{\delta}(\xi, \eta, p)= & \inf \int_{Y} F(\xi+\nabla w) d y, \\
& -\operatorname{div}\left(A \chi_{Z}+B \chi_{Y \backslash Z}\right)(\xi+\nabla w)=0 \text { in } \mathbf{R}^{N}, w \in H_{\sharp}^{1}(Y), \\
& \left|\int_{Y}\left(A \chi_{Z}+B \chi_{Y \backslash Z}\right)(\xi+\nabla w) d y-\eta\right|<\delta, \\
& Z \subset Y \text { measurable, }|Z|=p,
\end{aligned}\right.
$$

Copyright $@$ by SIAM. Unauthorized reproduction of this article is prohibited. 
for every $(\xi, \eta, p) \in \mathbf{R}^{N} \times \mathbf{R}^{N} \times[0,1]$. In the above expression, the infimum over the empty set is defined as $+\infty$.

By using the fact that $H_{\delta}$ is decreasing with respect to $\delta$, we define $H: \mathbf{R}^{N} \times$ $\mathbf{R}^{N} \times[0,1] \rightarrow \mathbf{R} \cup\{+\infty\}$ by

$$
H(\xi, \eta, p)=\lim _{\delta \rightarrow 0} H_{\delta}(\xi, \eta, p) \quad \forall(\xi, \eta, p) \in \mathbf{R}^{N} \times \mathbf{R}^{N} \times[0,1] .
$$

Remark 4.1. Definition (4.6) of $H$ implies that for every $(\xi, \eta, p) \in D(H)$ there exists a sequence of measurable sets $Z_{n} \subset Y$, with $\left|Z_{n}\right|=p$, such that by defining $S_{n} \in L^{\infty}\left(\Omega ; \mathcal{M}_{n}^{s}\right)$ by $S_{n}=A \chi_{Z_{n}}+B \chi_{Y \backslash Z_{n}}$, and taking the $w_{n} \in H_{\sharp}^{1}(Y)$ solution of

$$
-\operatorname{div} S_{n}\left(\xi+\nabla w_{n}\right)=0 \text { in } \mathbf{R}^{N}
$$

and

$$
\eta_{n}=\int_{Y} S_{n}\left(\xi+\nabla w_{n}\right) d y
$$

we have

$$
\begin{gathered}
\eta=\lim _{n \rightarrow \infty} \eta_{n}, \\
H(\xi, \eta, p)=\lim _{n \rightarrow \infty} \int_{Y} F\left(\xi+\nabla w_{n}\right) d y .
\end{gathered}
$$

For $N=1$, the following proposition gives an explicit expression of $H$.

Proposition 4.1. If $N=1$, the function $H$ is given by

$$
H(\xi, \eta, p)= \begin{cases}p F\left(\frac{\eta}{A}\right)+(1-p) F\left(\frac{\eta}{B}\right) & \text { if } \eta=\left(\frac{p}{A}+\frac{1-p}{B}\right)^{-1} \xi \\ +\infty & \text { in another case. }\end{cases}
$$

For $N \geq 2$ we do not have an explicit expression for $H$, but we can show the following result.

THEOREM 4.2. If $N \geq 2$, the function $H$ satisfies the following properties.

The domain of $H$ is given by

$$
\begin{aligned}
\operatorname{Dom}(H) & \stackrel{\text { def }}{=}\left\{(\xi, \eta, p) \in \mathbf{R}^{N} \times \mathbf{R}^{N} \times[0,1]: H(\xi, \eta, p)<+\infty\right\} \\
& =\left\{(\xi, \eta, p) \in \mathbf{R}^{N} \times \mathbf{R}^{N} \times[0,1]: \eta \in \mathcal{K}(A, B, p) \xi\right\} .
\end{aligned}
$$

The function $H$ is lower semicontinuous in $\operatorname{Dom}(H)$, and, for

$$
\alpha=\min \{\text { eigenvalues of } A \text { and } B\}, \quad \beta=\max \{\text { eigenvalues of } A \text { and } B\},
$$

it satisfies

$$
|H(\xi, \eta, p)| \leq L\left(\frac{\beta}{\alpha}|\xi|\right)^{\varrho}\left(1+\frac{\beta}{\alpha}|\xi|\right)^{2-\varrho} \quad \forall(\xi, \eta, p) \in \operatorname{Dom}(H) .
$$

Moreover, we have

$$
H(\xi, A \xi, 1)=H(\xi, B \xi, 0)=F(\xi) \quad \forall \xi \in \mathbf{R}^{N},
$$

Copyright (c) by SIAM. Unauthorized reproduction of this article is prohibited. 


$$
\left\{\begin{array}{l}
H(\xi, \eta, p)=p F\left(\xi+\Xi\left(\frac{\Lambda_{p} \xi-\eta}{p}\right)\right)+(1-p) F\left(\xi-\Xi\left(\frac{\Lambda_{p} \xi-\eta}{1-p}\right)\right) \\
\forall(\xi, \eta, p) \in \mathbf{R}^{N} \times \mathbf{R}^{N} \times(0,1) \text { with } \Lambda_{p} \xi-\eta \in \operatorname{Ran}(A-B) \text { and } \\
\left((A-B)\left(\frac{A}{p}+\frac{B}{1-p}\right)^{-1}(A-B)\right)^{\dagger}\left(\Lambda_{p} \xi-\eta\right) \cdot\left(\Lambda_{p} \xi-\eta\right)=\xi \cdot\left(\Lambda_{p} \xi-\eta\right) .
\end{array}\right.
$$

If $F$ is convex, then

$$
\begin{aligned}
F(\xi) & \leq \min \left\{p F\left(\xi+\frac{\nu}{p}\right)+(1-p) F\left(\xi-\frac{\nu}{1-p}\right):(B-A) \nu=\Lambda_{p} \xi-\eta, \nu \in E(\xi, p)\right\} \\
& \leq H(\xi, \eta, p) \quad \forall(\xi, \eta, p) \in \operatorname{Dom}(H), p \in(0,1) .
\end{aligned}
$$

Remark 4.2. A consequence of Theorem 4.2 is that if $N \geq 2$, then $H\left(\xi, \Lambda_{p} \xi, p\right)=$ $F(\xi)$ for every $\xi \in \mathbf{R}^{N}$ and every $p \in[0,1]$.

Remark 4.3. If $(\xi, \eta, p) \in \mathbf{R}^{N} \times \mathbf{R}^{N} \times(0,1)$ is such that $\Lambda_{p} \xi-\eta \in \operatorname{Ran}(A-B)$ and

$$
\left((A-B)\left(\frac{A}{p}+\frac{B}{1-p}\right)^{-1}(A-B)\right)^{\dagger}\left(\Lambda_{p} \xi-\eta\right) \cdot\left(\Lambda_{p} \xi-\eta\right)=\xi \cdot\left(\Lambda_{p} \xi-\eta\right)
$$

then the set of $\nu \in E(\xi, p)$ such that $(B-A) \nu=\Lambda_{p} \xi-\eta$ reduces to $\nu=\Xi\left(\Lambda_{p} \xi-\eta\right)$. Therefore, (4.13) shows that the second inequality in (4.14) is an equality for such $(\xi, \eta, p)$.

By using the function $H$, we obtain the following theorem.

TheOREM 4.3. For every $G: H_{0}^{1}(\Omega) \rightarrow \mathbf{R}$ sequentially continuous in the weak topology of $H_{0}^{1}(\Omega)$, every $\kappa \in(0,1)$, and every $f \in H^{-1}(\Omega)$, a relaxation of problem (4.4) is given by

$$
\left\{\begin{array}{l}
\min \left\{\int_{\Omega} H(\nabla u, M \nabla u, \theta) d x+G(u)\right\}, \\
-\operatorname{div} M \nabla u=f \text { in } \Omega, \\
u \in H_{0}^{1}(\Omega), \\
\theta \in L^{\infty}(\Omega), 0 \leq \theta \leq 1 \text { a.e. in } \Omega, \int_{\Omega} \theta d x \leq \kappa|\Omega|, \\
M \text { measurable, } M(x) \in \mathcal{K}(A, B, \theta(x)) \text { for a.e. } x \in \Omega,
\end{array}\right.
$$

with $H$ given by (4.6).

Remark 4.4. Problem (4.16) can also be written as

$$
\left\{\begin{array}{l}
\min \left\{\int_{\Omega} H(\nabla u, \sigma, \theta) d x+G(u)\right\}, \\
-\operatorname{div} \sigma=f \text { in } \Omega, \\
u \in H_{0}^{1}(\Omega), \\
\theta \in L^{\infty}(\Omega), 0 \leq \theta \leq 1 \text { a.e. in } \Omega, \int_{\Omega} \theta d x \leq \kappa|\Omega|, \\
\sigma \in \mathcal{K}(A, B, \theta) \nabla u \text { a.e. in } \Omega \text { measurable. }
\end{array}\right.
$$

Copyright $@$ ( ) by SIAM. Unauthorized reproduction of this article is prohibited. 
Theorem 4.3 is a consequence of Theorem 4.5 below, which is interesting by itself. We need the following definition.

DeFinition 4.4. We say that $\left(u_{n}, \sigma_{n}, \theta_{n}\right) \in H^{1}(\Omega) \times L^{2}(\Omega)^{N} \times L^{\infty}(\Omega) \mathcal{T}$. converges to $(u, \sigma, \theta) \in H^{1}(\Omega) \times L^{2}(\Omega)^{N} \times L^{\infty}(\Omega)$ if and only if

$$
\begin{array}{ll}
u_{n} \rightarrow \text { in } H^{1}(\Omega), & \left|\nabla u_{n}\right|^{2} \text { equi-integrable, } \\
\sigma_{n} \rightarrow \sigma \text { in } L^{2}(\Omega)^{N}, & \operatorname{div} \sigma_{n} \rightarrow \operatorname{div} \sigma \text { in } H^{-1}(\Omega)^{N}, \\
\theta_{n} \stackrel{*}{\rightarrow} \text { in } L^{\infty}(\Omega) . &
\end{array}
$$

Remark 4.5. In the applications, we are interested in sequences $\left(u_{n}, \sigma_{n}, \theta_{n}\right)$ such that there exists a sequence of uniformly elliptic and bounded matrix functions $M_{n}$, which satisfies $M_{n} \nabla u_{n}=\sigma_{n}$. Then we recall that, thanks to Meyer's regularity theorem [19], the weak convergence of $u_{n}$ in $H^{1}(\Omega)$ and the strong convergence of $\sigma_{n}$ in $L^{2}(\Omega)^{N}$ imply the equi-integrability of $\left|\nabla u_{n}\right|^{2}$ at least for $\Omega$ smooth and $u_{n}$ satisfying "good" boundary conditions (if not, we always hold the equi-integrability on compact subsets of $\Omega$ ).

THEOREM 4.5. The lower semicontinuous envelope with respect to the $\mathcal{T}$ convergence of the functional $\mathcal{F}: H^{1}(\Omega) \times L^{2}(\Omega)^{N} \times L^{\infty}(\Omega) \rightarrow \mathbf{R} \cup\{+\infty\}$ defined by

$$
\mathcal{F}(u, \sigma, \theta)= \begin{cases}\int_{\Omega} F(\nabla u) d x & \text { if } \theta=\chi_{\omega}, \omega \subset \Omega \text { measurable, } \sigma=\Lambda_{\theta} \nabla u \\ +\infty & \text { in another case }\end{cases}
$$

is given by

$$
\overline{\mathcal{F}}(u, \sigma, \theta)= \begin{cases}\int_{\Omega} H(\nabla u, \sigma, \theta) d x & \text { if } 0 \leq \theta \leq 1, \sigma \in \mathcal{K}(A, B, \theta) \nabla u, \text { a.e. in } \Omega, \\ +\infty & \text { in another case. }\end{cases}
$$

Remark 4.6. Analogously to the proof of Theorem 4.3, we can use Theorem 4.5 to obtain the relaxation of some other related control problems. For example (assuming smoothness enough to have the equi-integrability of $\left|\nabla u_{n}\right|^{2}$, with $u_{n}$ the state functions corresponding to a minimizing sequence), we can consider different boundary conditions for the state equation and some other restrictions. In this way, we can apply Theorem 4.5 to obtain a relaxation of the control problem defining $H_{\delta}$ (see (4.5)). This permits us to prove that the function $H$ given by (4.6) satisfies

$$
\left\{\begin{aligned}
H(\xi, \eta, p)= & \inf \int_{Y} H(\xi+\nabla w, M(\xi+\nabla w), \theta) d y \\
& \theta \in L_{\sharp}^{\infty}(Y), \quad 0 \leq \theta \leq 1 \text { a.e. in } \mathbf{R}^{N}, \quad \int_{Y} \theta d y=p, \\
& M \in \mathcal{K}(A, B, \theta) \text { a.e. in } \mathbf{R}^{N}, \quad w \in H_{\sharp}^{1}(Y), \\
& -\operatorname{div} M(\xi+\nabla w)=0 \text { in } \mathbf{R}^{N}, \\
& \int_{Y} M(\xi+\nabla w) d y=\eta .
\end{aligned}\right.
$$

Copyright $@$ by SIAM. Unauthorized reproduction of this article is prohibited. 
Remark 4.7. In Step 3 in the proof of Theorem 4.5, for $(u, \sigma, \theta) \in \operatorname{Dom}(\overline{\mathcal{F}})$ given, we show how to construct $\omega_{n} \subset \Omega$ and $u_{n} \in H^{1}\left(\Omega_{n}\right)$ such that the sequence $\left(u_{n},\left(A \chi_{\omega_{n}}+B \chi_{\omega_{n}}\right) \nabla u_{n}, \chi_{\omega_{n}}\right) \in \operatorname{Dom}(\mathcal{F})$ satisfies

$$
\overline{\mathcal{F}}(u, \sigma, \theta)=\lim _{n \rightarrow \infty} \mathcal{F}\left(u_{n},\left(A \chi_{\omega_{n}}+B \chi_{\omega_{n}}\right) \nabla u_{n}, \chi_{\omega_{n}}\right) .
$$

By applying this procedure to a solution $(u, \sigma, \theta)$ of problem (4.17), this gives a way to construct a minimizing sequence for problem (4.4). Unfortunately, to apply this procedure it is necessary, for $(\xi, \eta, p) \in \operatorname{Dom}(H)$, to know how to construct $Z_{n} \subset Y$ in the conditions of Remark 4.1. We do not know how to make this, in general. In particular, we do not know if this can be carried out by using laminations, as it happens in some particular cases where the function $H$ can be explicitly calculated (see, e.g., [3], [10], and Remark 6.2 in the present paper). When $\eta \in \partial \mathcal{K}(A, B, p) \xi$ (here $\partial$ denotes the boundary with respect to the affine hull), the set $Z_{n}$ is obtained by using a simple lamination in the direction of $\nu=\Xi\left(\Lambda_{p} \xi-\eta\right)$, if $\eta \neq \Lambda_{p} \xi$, or in an orthogonal direction to $(B-A) \xi$, if $\eta=\Lambda_{p} \xi$. In this sense, we remark that if $H$ is derivable (which we do not know if it is true) and $\theta, M, u$ is a solution of (4.16), then, by introducing the adjoint state $q$ as the solution of

$$
\left\{\begin{array}{l}
-\operatorname{div} M \nabla q=-\operatorname{div}\left(\nabla_{\xi} H(\nabla u, M \nabla u, \theta)+M \nabla_{\eta} H(\nabla u, M \nabla u, \theta)\right) \text { in } \Omega, \\
q \in H_{0}^{1}(\Omega),
\end{array}\right.
$$

the optimality conditions for problem (4.16) show (see, e.g., [1], [5], [16], [24] for related results) that a.e. on the set $\left\{x \in \Omega: \nabla q(x) \neq \nabla_{\eta} H(\nabla u(x), M(x) \nabla u(x), \theta(x))\right\}$, one has that $M \nabla u \in \partial \mathcal{K}(A, B, \theta) \nabla u$.

Remark 4.8. In order to solve numerically problem (4.16) the main difficulty is, as in the previous remark, that we have only an explicit expression of $H$ on the points $(\xi, \eta, p)$ such that $\eta \in \partial \mathcal{K}(A, B, p) \xi$ (see (4.13)). But, as we observed above, if $\theta, M$, $u$ is a solution of (4.16), $H$ is sufficiently smooth, and $\nabla q \neq \nabla_{\eta} H(\nabla u, M \nabla u, \theta)$ a.e. in $\Omega$, then $M \nabla u \in \partial \mathcal{K}(A, B, \theta) \nabla u$ a.e. in $\Omega$. Moreover, in this case $M$ is obtained by just one lamination. By taking into account these remarks, one can consider a numerical method consisting, for example, of taking a triangulation of $\Omega$ and then searching the state function $u$ piecewise affine, the proportion $\theta$, and the matrix $M$ piecewise constants, with $M$ corresponding to a lamination in each triangle (so the choice of $M$ in each triangle is reduced to the choice of the corresponding lamination vector). This provides a numerical method similar to the one used in [15] and [31] for the case where $F(\xi)=|\xi|^{2}, A=\alpha I, B=\beta I, \alpha, \beta>0$.

By using the fact that by Theorem 4.5 the functional $\overline{\mathcal{F}}$ is lower semicontinuous for the $\mathcal{T}$-convergence, we can deduce some convexity properties for $H$. The result is essentially a consequence of the compensated compactness theory of Murat [23] and Tartar [28].

Proposition 4.6. The function $H$ defined by (4.6) satisfies the following convexity properties:

(i) If $N=1$, then

$$
H\left(\lambda \xi_{1}+(1-\lambda) \xi_{2}, \eta, \lambda p_{1}+(1-\lambda) p_{2}\right) \leq \lambda H\left(\xi_{1}, \eta, p_{1}\right)+(1-\lambda) H\left(\xi_{2}, \eta, p_{2}\right)
$$

for every $\xi_{1}, \xi_{2}, \eta \in \mathbf{R}$ and every $p_{1}, p_{2}, \lambda \in[0,1]$.

(ii) If $N \geq 2$, then

$$
\begin{aligned}
& H\left(\lambda \xi_{1}+(1-\lambda) \xi_{2}, \lambda \eta_{1}+(1-\lambda) \eta_{2}, \lambda p_{1}+(1-\lambda) p_{2}\right) \\
& \leq \lambda H\left(\xi_{1}, \eta_{1}, p_{1}\right)+(1-\lambda) H\left(\xi_{2}, \eta_{2}, p_{2}\right)
\end{aligned}
$$

Copyright (c) by SIAM. Unauthorized reproduction of this article is prohibited. 
for every $\xi_{1}, \xi_{2}, \eta_{1}, \eta_{2} \in \mathbf{R}^{N}$, with $\left(\xi_{2}-\xi_{1}\right) \cdot\left(\eta_{2}-\eta_{1}\right)=0$, and every $p_{1}, p_{2}, \lambda \in[0,1]$.

As a consequence of this result we will prove the following proposition which improves Theorem 4.2 .

Proposition 4.7. The function $H$ is continuous on its domain.

Remark 4.9. In Theorem 4.2, we gave a lower bound for $H$ by assuming $F$ convex. An analogous proof shows that for $F$ concave we have

$$
\begin{aligned}
& H(\xi, \eta, p) \\
& \quad \leq \max \left\{p F\left(\xi+\frac{\nu}{p}\right)+(1-p) F\left(\xi-\frac{\nu}{1-p}\right):(B-A) \nu=\Lambda_{p} \xi-\eta, \nu \in E(\xi, p)\right\}
\end{aligned}
$$

for every $(\xi, \eta, p) \in \operatorname{Dom}(H)$. Indeed, since $H$ is defined by a minimum, it is not difficult to obtain upper bounds for $H$. In this way, by using (4.13), (4.22), and $H$ lower semicontinuous for the $\mathcal{T}$-convergence, we can use the reasoning at the end of the proof of Proposition 3.3 to show that for every $F$ satisfying (4.1) and (4.2) (not necessarily concave), every $(\xi, \eta, p) \in \operatorname{Dom}(H), p \in(0,1)$, and every $\nu \in E(\xi, p)$, with $(B-A) \nu=\Lambda_{p} \xi-\eta$, we have

$$
H(\xi, \eta, p) \leq\left(1-\frac{1}{\lambda}\right) F(\xi)+\frac{1}{\lambda}\left(p F\left(\xi+\frac{\lambda \nu}{p}\right)+(1-p) F\left(\xi-\frac{\lambda \nu}{1-p}\right)\right),
$$

where

$$
\lambda=\frac{\left(\Lambda_{p} \xi-\eta\right) \cdot \xi}{\left(\frac{A}{p}+\frac{B}{1-p}\right) \nu \cdot \nu} \in[0,1] .
$$

In particular, for $F$ concave, this proves that

$$
\begin{aligned}
& H(\xi, \eta, p) \\
& \quad \leq \min \left\{p F\left(\xi+\frac{\nu}{p}\right)+(1-p) F\left(\xi-\frac{\nu}{1-p}\right):(B-A) \nu=\Lambda_{p} \xi-\eta, \nu \in E(\xi, p)\right\} .
\end{aligned}
$$

5. Proofs of the results of section 4. Throughout this section, for a measurable set $Z \subset Y$ and $\xi \in \mathbf{R}^{N}$, we usually associate a matrix function $S$, defined by

$$
S=A \chi_{Z}+B \chi_{Y \backslash Z},
$$

and a function $w$ solution of (3.2).

Proof of Proposition 4.1. The result is a simple consequence of Remark 4.1 and the fact that the solution $w$ of $(3.2)$ with $|Z|=p \in[0,1]$ satisfies (3.9), with $\eta$ given by $(3.10)$.

In order to prove Theorem 4.2, we first obtain some bounds for $\nabla w$, with the $w$ solution of (3.2). This will be done in Lemmas 5.1 and 5.2 below.

Lemma 5.1. For every $Z \subset Y$ measurable and $\xi \in \mathbf{R}^{N}$, the function $w$ satisfies

$$
\|\xi+\nabla w\|_{L^{2}(Y)^{N}} \leq \frac{\beta}{\alpha}|\xi| .
$$

Moreover, there exist $r>2$ and $C>0$, which depend only on $\beta / \alpha$ and $N$, such that $w \in W_{\sharp}^{1, r}(Y)$ and

$$
\|\xi+\nabla w\|_{L^{r}(Y)^{N}} \leq C|\xi|
$$

Copyright $@$ by SIAM. Unauthorized reproduction of this article is prohibited. 
Proof. The proof of (5.2) easily follows by using $w$ as a test function in (3.2). Estimate (5.3) is a consequence of Meyer's regularity theorem [19] and (5.2).

Lemma 5.2. There exist $C>0$ and $\rho \in(0,1)$ (which depend only on $\beta / \alpha$ and $N$ ) such that, for every $\xi, \xi^{\prime} \in \mathbf{R}^{N}$ and every $Z, Z^{\prime} \subset Y$ measurable, the corresponding functions $S, S^{\prime}, w, w^{\prime}$ satisfy

$$
\begin{gathered}
\left\|\xi+\nabla w-\xi^{\prime}-\nabla w^{\prime}\right\|_{L^{2}(Y)^{N}} \leq C\left(\left|\xi-\xi^{\prime}\right|+\left|\xi^{\prime}\right|\left|Z \triangle Z^{\prime}\right|^{\rho}\right), \\
\left|\int_{Y} S(\xi+\nabla w) d y-\int_{Y} S^{\prime}\left(\xi^{\prime}+\nabla w^{\prime}\right) d y\right| \leq C\left(\left|\xi-\xi^{\prime}\right|+\left|\xi^{\prime}\right|\left|Z \triangle Z^{\prime}\right|^{\rho}\right) .
\end{gathered}
$$

Proof. By taking $w-w^{\prime}$ as a test function in the difference of the equations satisfied by $w$ and $w^{\prime}$ and adding and subtracting convenient terms, we get

$$
\begin{aligned}
& \int_{Y} S\left(\xi+\nabla w-\xi^{\prime}-\nabla w^{\prime}\right) \cdot\left(\xi+\nabla w-\xi^{\prime}-\nabla w^{\prime}\right) d x \\
& =\int_{Y}\left(S^{\prime}-S\right)\left(\xi^{\prime}+\nabla w^{\prime}\right) \cdot\left(\xi+\nabla w-\xi^{\prime}-\nabla w^{\prime}\right) d y \\
& +\int_{Y} S\left(\xi+\nabla w-\xi^{\prime}-\nabla w^{\prime}\right) \cdot\left(\xi-\xi^{\prime}\right) d y-\int_{Y}\left(S^{\prime}-S\right)\left(\xi^{\prime}+\nabla w^{\prime}\right) \cdot\left(\xi-\xi^{\prime}\right) d y .
\end{aligned}
$$

By using the ellipticity of $S$, Young's inequality, and $S=S^{\prime}$ in $Y \backslash\left(Z \triangle Z^{\prime}\right)$, we obtain

$$
\int_{Y}\left|\xi+\nabla w-\xi^{\prime}-\nabla w^{\prime}\right|^{2} d y \leq C\left(\int_{Z \triangle Z^{\prime}}\left|\xi^{\prime}+\nabla w^{\prime}\right|^{2} d y+\left|\xi-\xi^{\prime}\right|^{2}\right),
$$

where $C$ depends only on $\beta / \alpha$. The first term on the right-hand side of this inequality can be estimated by (5.3), which gives

$$
\int_{Z \triangle Z^{\prime}}\left|\xi^{\prime}+\nabla w^{\prime}\right|^{2} d y \leq\left(\int_{Y}\left|\xi^{\prime}+\nabla w^{\prime}\right|^{r} d y\right)^{\frac{2}{r}}\left|Z \triangle Z^{\prime}\right|^{1-\frac{2}{r}} \leq C\left|\xi^{\prime}\right|^{2}\left|Z \triangle Z^{\prime}\right|^{1-\frac{2}{r}} .
$$

By substituting (5.7) into (5.6) we get (5.4).

In order to prove (5.5) we now use

$$
\begin{aligned}
& \left|\int_{Y} S(\xi+\nabla w) d y-\int_{Y} S^{\prime}\left(\xi^{\prime}+\nabla w^{\prime}\right) d y\right| \\
& \leq\left|\int_{Y} S\left(\xi+\nabla w-\xi^{\prime}-\nabla w^{\prime}\right) d y\right|+\left|\int_{Y}\left(S-S^{\prime}\right)\left(\xi^{\prime}+\nabla w^{\prime}\right) d y\right| \\
& \leq C\left(\int_{Y}\left|\xi+\nabla w-\xi^{\prime}-\nabla w^{\prime}\right| d y+\int_{Z \triangle Z^{\prime}}\left|\xi^{\prime}+\nabla w^{\prime}\right| d y\right) .
\end{aligned}
$$

By using the Cauchy-Schwarz inequality, (5.4), and (5.7), we obtain (5.5).

Let us now use Lemma 5.2 to study some semicontinuity properties for $H_{\delta}$.

Lemma 5.3. There exist $C>0$ (depending on $L, \beta / \alpha, \varrho$, and $N$ ) and $\rho \in(0,1)$ (depending on $\beta / \alpha$ and $N$ ) such that, for every $(\xi, \eta, p) \in \mathbf{R}^{N} \times \mathbf{R}^{N} \times[0,1]$ and every $\delta, \varepsilon>0$, there exists $\tau \in[0, \delta)$, with

$$
\begin{aligned}
& H_{\tau+\lambda^{\prime}+\left|\eta-\eta^{\prime}\right|}\left(\xi^{\prime}, \eta^{\prime}, p^{\prime}\right) \leq H_{\delta}(\xi, \eta, p)+\left(1+|\xi|+\left|\xi^{\prime}\right|\right)^{2-\varrho}\left(\lambda^{\prime}\right)^{\varrho}+\varepsilon \\
& \forall\left(\xi^{\prime}, \eta^{\prime}, p^{\prime}\right) \in \mathbf{R}^{N} \times \mathbf{R}^{N} \times[0,1],
\end{aligned}
$$

Copyright $@$ by SIAM. Unauthorized reproduction of this article is prohibited. 
where we have denoted

$$
\lambda^{\prime}=C\left(\left|\xi-\xi^{\prime}\right|+\left|\xi^{\prime}\right|\left|p-p^{\prime}\right|^{\rho}\right) .
$$

Proof. To prove (5.8), we consider $(\xi, \eta, p) \in \mathbf{R}^{N} \times \mathbf{R}^{N} \times[0,1]$ and $\delta, \varepsilon>0$. If $(\xi, \eta, p)$ does not belong to the domain of $H_{\delta}$, then (5.8) is trivial. In another case, by definition (4.5) of $H_{\delta}$ there exists $Z \subset Y$, with $|Z|=p$, such that, by taking $S$ and $w$ as the corresponding functions associated to $Z$ and $\xi$ and defining $\tau$ by

$$
\tau=\left|\int_{Y} S(\xi+\nabla w) d y-\eta\right|
$$

we have $\tau<\delta$ and

$$
H_{\delta}(\xi, \eta, p)>\int_{Y} F(\xi+\nabla w) d y-\varepsilon .
$$

Now we consider $\left(\xi^{\prime}, \eta^{\prime}, p^{\prime}\right) \in \mathbf{R}^{N} \times \mathbf{R}^{N} \times[0,1]$. Then (by adding or subtracting a measurable set of $Y$ to $Z$ ) we construct a set $Z^{\prime}$, with $\left|Z^{\prime}\right|=p^{\prime}$ and $\left|Z \triangle Z^{\prime}\right|=\left|p-p^{\prime}\right|$. We take $w^{\prime}$ and $S^{\prime}$ as the corresponding functions associated to $Z^{\prime}$ and $\xi^{\prime}$. From Lemma 5.2, there exist $C>0$ and $\rho \in(0,1)$, which depend only on $\beta / \alpha$ and $N$, such that (5.4) and (5.5) hold. By using then (4.1), (5.2), and (5.4), we have (for another constant $C$ )

$$
\begin{aligned}
& \left|\int_{Y}\left(F(\xi+\nabla w)-F\left(\xi^{\prime}+\nabla w^{\prime}\right)\right) d y\right| \\
& \leq L \int_{Y}\left(1+|\xi+\nabla w|+\left|\xi^{\prime}+\nabla w^{\prime}\right|\right)^{2-\varrho}\left|\xi+\nabla w-\xi^{\prime}-\nabla w^{\prime}\right|^{\varrho} d y \\
& \leq L\left(1+\|\xi+\nabla w\|_{L^{2}(Y)}+\left\|\xi^{\prime}+\nabla w^{\prime}\right\|_{L^{2}(Y)}\right)^{2-\varrho}\left\|\xi+\nabla w-\xi^{\prime}-\nabla w^{\prime}\right\|_{L^{2}(Y)}^{\varrho} \\
& \leq C\left(1+|\xi|+\left|\xi^{\prime}\right|\right)^{2-\varrho}\left(\left|\xi-\xi^{\prime}\right|+\left|\xi^{\prime}\right|\left|p-p^{\prime}\right|^{\rho}\right)^{\varrho} \leq\left(1+|\xi|+\left|\xi^{\prime}\right|\right)^{2-\varrho}\left(\lambda^{\prime}\right)^{\varrho} .
\end{aligned}
$$

On the other hand, by (5.5) and the definition of $\tau$, we have

$$
\begin{aligned}
& \left|\int_{Y} S^{\prime}\left(\xi^{\prime}+\nabla w^{\prime}\right) d y-\eta^{\prime}\right| \leq\left|\int_{Y} S^{\prime}\left(\xi^{\prime}+\nabla w^{\prime}\right) d y-\int_{Y} S(\xi+\nabla w) d y\right| \\
& +\left|\int_{Y} S(\xi+\nabla w) d y-\eta\right|+\left|\eta-\eta^{\prime}\right| \leq \lambda^{\prime}+\tau+\left|\eta-\eta^{\prime}\right| .
\end{aligned}
$$

Then, by definition (4.5) of $H_{\delta}$, we get

$$
\begin{aligned}
& H_{\tau+\lambda^{\prime}+\left|\eta-\eta^{\prime}\right|}\left(\xi^{\prime}, \eta^{\prime}, p^{\prime}\right) \leq \int_{Y} F\left(\xi^{\prime}+\nabla w^{\prime}\right) d y \\
& \leq \int_{Y} F(\xi+\nabla w) d y+\left|\int_{Y}\left(F(\xi+\nabla w)-F\left(\xi^{\prime}+\nabla w^{\prime}\right)\right) d y\right| \\
& \leq H_{\delta}(\xi, \eta, p)+\varepsilon+\left(1+|\xi|+\left|\xi^{\prime}\right|\right)^{2-\varrho}\left(\lambda^{\prime}\right)^{\varrho} .
\end{aligned}
$$

Remark 5.1. Since $H_{\delta}(\xi, \eta, p)$ is decreasing in $\delta$ inequality (5.8) implies that

$$
\begin{aligned}
& H_{\delta+\lambda^{\prime}+\mid \eta-\eta^{\prime}}\left(\xi^{\prime}, \eta^{\prime}, p^{\prime}\right) \leq H_{\delta}(\xi, \eta, p)+\left(1+|\xi|+\left|\xi^{\prime}\right|\right)^{2-\varrho}\left(\lambda^{\prime}\right)^{\varrho}+\varepsilon \\
& \forall\left(\xi^{\prime}, \eta^{\prime}, p^{\prime}\right) \in \mathbf{R}^{N} \times \mathbf{R}^{N} \times[0,1]
\end{aligned}
$$

Copyright (c) by SIAM. Unauthorized reproduction of this article is prohibited. 
for every $\varepsilon>0$. So by taking $\varepsilon$ converging to zero we get

$$
\begin{aligned}
& H_{\delta+\lambda^{\prime}+\left|\eta-\eta^{\prime}\right|}\left(\xi^{\prime}, \eta^{\prime}, p^{\prime}\right) \leq H_{\delta}(\xi, \eta, p)+\left(1+|\xi|+\left|\xi^{\prime}\right|\right)^{2-\varrho}\left(\lambda^{\prime}\right)^{\varrho} \\
& \forall\left(\xi^{\prime}, \eta^{\prime}, p^{\prime}\right) \in \mathbf{R}^{N} \times \mathbf{R}^{N} \times[0,1] .
\end{aligned}
$$

We are now in position to prove that $H_{\delta}$ satisfies the following properties.

Proposition 5.4. For every $\delta>0, H_{\delta}$ is upper semicontinuous in $\mathbf{R}^{N} \times \mathbf{R}^{N} \times$ $[0,1]$ and satisfies

$$
\left|H_{\delta}(\xi, \eta, p)\right| \leq L\left(\frac{\beta}{\alpha}|\xi|\right)^{\varrho}\left(1+\frac{\beta}{\alpha}|\xi|\right)^{2-\varrho} \quad \forall(\xi, \eta, p) \in \operatorname{Dom}\left(H_{\delta}\right) .
$$

Moreover, the following lower semicontinuity result holds:

$$
H_{\delta+s}(\xi, \eta, p) \leq \liminf _{n \rightarrow \infty} H_{\delta}\left(\xi_{n}, \eta_{n}, p_{n}\right) \quad \forall \delta, s>0
$$

for every $(\xi, \eta, p) \in \mathbf{R}^{N} \times \mathbf{R}^{N} \times[0,1]$ and for every sequence $\left(\xi_{n}, \eta_{n}, p_{n}\right) \in \mathbf{R}^{N} \times$ $\mathbf{R}^{N} \times[0,1]$ which converges to $(\xi, \eta, p)$.

Proof. The proof of (5.10) immediately follows from definition (4.5) of $H_{\delta}$, by taking into account that (5.2), (4.2), and (4.1) imply that for every $\xi \in \mathbf{R}^{N}$ and every $Z \subset Y$ measurable the solution $w$ of (3.2) satisfies

$$
\left|\int_{Y} F(\xi+\nabla w) d y\right| \leq L\left(\frac{\beta}{\alpha}|\xi|\right)^{\varrho}\left(1+\frac{\beta}{\alpha}|\xi|\right)^{2-\varrho} .
$$

To prove the upper semicontinuity of $H$ we consider $(\xi, \eta, p)$ and $\left(\xi_{n}, \eta_{n}, p_{n}\right)$ as above. By (5.8), for every $\delta, \varepsilon>0$, there exists $\tau \in[0, \delta$ ) (which does not depend on $n$ ) such that

$$
H_{\tau+\lambda_{n}+\left|\eta-\eta_{n}\right|}\left(\xi_{n}, \eta_{n}, p_{n}\right) \leq H_{\delta}(\xi, \eta, p)+\left(1+|\xi|+\left|\xi_{n}\right|\right)^{2-\varrho} \lambda_{n}^{\varrho}+\varepsilon \quad \forall n \in \mathbf{N},
$$

with $\lambda_{n}=C\left(\left|\xi-\xi_{n}\right|+\left|\xi_{n}\right|\left|p-p_{n}\right|^{\rho}\right)$. So, since for $n$ large enough $\tau+\lambda_{n}+\left|\eta-\eta_{n}\right|<\delta$ and $H_{\mu}$ is decreasing in $\mu$, we have

$$
H_{\delta}\left(\xi_{n}, \eta_{n}, p_{n}\right) \leq H_{\delta}(\xi, \eta, p)+\left(1+|\xi|+\left|\xi_{n}\right|\right)^{2-\varrho} \lambda_{n}^{\varrho}+\varepsilon .
$$

By taking the limsup in $n$ and then letting $\varepsilon$ decrease to zero, we deduce the upper semicontinuity of $H_{\delta}$.

In order to prove $(5.11)$, we take $(\xi, \eta, p),\left(\xi_{n}, \eta_{n}, p_{n}\right)$ as above. By (5.9) we have

$$
H_{\delta+\lambda_{n}+\left|\eta-\eta_{n}\right|}(\xi, \eta, p) \leq H_{\delta}\left(\xi_{n}, \eta_{n}, p_{n}\right)+\left(1+|\xi|+\left|\xi_{n}^{\prime}\right|\right)^{2-\varrho} \lambda_{n}^{\varrho},
$$

with $\lambda_{n}=C\left(\left|\xi-\xi_{n}\right|+|\xi|\left|p-p_{n}\right|^{\rho}\right)$. So, by using as above the fact that $H_{\mu}$ is decreasing in $\mu$, we have for every $s>0$ and $n$ large enough

$$
H_{\delta+s}(\xi, \eta, p) \leq H_{\delta}\left(\xi_{n}, \eta_{n}, p_{n}\right)+\left(1+|\xi|+\left|\xi_{n}^{\prime}\right|\right)^{2-\varrho} \lambda_{n}^{\varrho} .
$$

By taking the liminf in $n$ we deduce (5.11).

Copyright (c) by SIAM. Unauthorized reproduction of this article is prohibited. 
Thanks to the previous results, we can now prove Theorem 4.2.

Proof of Theorem 4.2. By definition (4.6) of $H$ and (5.12), we have that $(\xi, \eta, p) \in$ $\operatorname{Dom}(H)$ if and only if for every $\delta>0$ there exists $Z \subset Y$ measurable, with $|Z|=p$, such that the solution $w$ of (3.2) satisfies

$$
\left|\int_{Y} S(\nabla w+\xi) d y-\eta\right|<\delta .
$$

By Theorem 3.1 this holds if and only if $\eta$ belongs to $\mathcal{K}(A, B, p) \xi$. This proves (4.10).

In order to prove the lower semicontinuity of $H$, we consider $(\xi, \eta, p) \in \mathbf{R}^{N} \times$ $\mathbf{R}^{N} \times[0,1]$ and $\left(\xi_{n}, \eta_{n}, p_{n}\right) \in \mathbf{R}^{N} \times \mathbf{R}^{N} \times[0,1]$, which converges to $(\xi, \eta, p)$. By (5.11) with $s=\delta$ and $H_{\delta}\left(\xi_{n}, \eta_{n}, p_{n}\right) \leq H\left(\xi_{n}, \eta_{n}, p_{n}\right)$, we have

$$
H_{2 \delta}(\xi, \eta, p) \leq \liminf _{n \rightarrow \infty} H\left(\xi_{n}, \eta_{n}, p_{n}\right) \quad \forall \delta>0 .
$$

By taking the limit when $\delta$ tends to zero we conclude that

$$
H(\xi, \eta, p) \leq \liminf _{n \rightarrow \infty} H\left(\xi_{n}, \eta_{n}, p_{n}\right)
$$

and then the lower semicontinuity of $H$.

Inequality (4.11) immediately follows from (5.10).

To show (4.12) it is enough to use Remark 4.1 and the fact that, if $Z \subset Y$ has measure 0 or 1 , the solution $w$ of (3.2) is zero.

To prove (4.13), we consider $(\xi, \eta, p) \in \mathbf{R}^{N} \times \mathbf{R}^{N} \times(0,1)$ such that $\Lambda_{p} \xi-\eta \in$ $\operatorname{Ran}(A-B)$ and

$$
\left((A-B)\left(\frac{A}{p}+\frac{B}{1-p}\right)^{-1}(A-B)\right)^{\dagger}\left(\Lambda_{p} \xi-\eta\right) \cdot\left(\Lambda_{p} \xi-\eta\right)=\xi \cdot\left(\Lambda_{p} \xi-\eta\right) .
$$

Then we consider $Z_{n}, S_{n}, w_{n}, \eta_{n}$ as in Remark 4.1, and we define

$$
\nu_{n}=\int_{Z_{n}} \nabla w_{n} d y=-\int_{Y \backslash Z_{n}} \nabla w_{n} d y .
$$

By (3.5) and (3.6), $\nu_{n}$ satisfies that $(B-A) \nu_{n}=\Lambda_{p} \xi-\eta_{n}$ and

$$
\left\{\begin{array}{l}
\int_{Z_{n}} A\left(\nabla w_{n}-\frac{\nu_{n}}{p}\right) \cdot\left(\nabla w_{n}-\frac{\nu_{n}}{p}\right) d y \\
+\int_{Y \backslash Z_{n}} B\left(\nabla w_{n}+\frac{\nu_{n}}{1-p}\right) \cdot\left(\nabla w_{n}+\frac{\nu_{n}}{1-p}\right) d y \\
=\left(\Lambda_{p} \xi-\eta_{n}\right) \cdot \xi-\left(\frac{A}{p}+\frac{B}{1-p}\right) \nu_{n} \cdot \nu_{n} .
\end{array}\right.
$$

This implies in particular (use the fact that the left-hand side of (5.14) is nonnegative) that $\nu_{n}$ is bounded, and so, up to a subsequence, we have that $\nu_{n}$ converges to some $\hat{\nu} \in \mathbf{R}^{N}$ such that $(B-A) \hat{\nu}=\Lambda_{p} \xi-\eta$ and

$$
\left(\frac{A}{p}+\frac{B}{1-p}\right) \hat{\nu} \cdot \hat{\nu} \leq\left(\Lambda_{p} \xi-\eta\right) \cdot \xi .
$$

Copyright $@$ by SIAM. Unauthorized reproduction of this article is prohibited. 
By using the fact that (5.13) can also be written as

$$
\left(\Lambda_{p} \xi-\eta\right) \cdot \xi=\min \left\{\left(\frac{A}{p}+\frac{B}{1-p}\right) \nu \cdot \nu: \Lambda_{p} \xi-\eta=(B-A) \nu\right\},
$$

we then deduce that $\hat{\nu}$ gives the minimum above, and so $\hat{\nu}=\Xi\left(\Lambda_{p} \xi-\eta\right)$. In particular, this implies that (5.15) is an equality, and then by passing to the limit in (5.14) we have

$$
\lim _{n \rightarrow 0}\left(\int_{Z_{n}}\left|\nabla w_{n}-\frac{\nu_{n}}{p}\right|^{2} d z+\int_{Y \backslash Z_{n}}\left|\nabla w_{n}+\frac{\nu_{n}}{1-p}\right|^{2} d z\right)=0,
$$

which, joining to (4.1), allows us to calculate the limit which appears in the right-hand side of (4.8) and then to conclude (4.13).

To finish the proof of Theorem 4.2, let us now prove (4.14). For $(\xi, \eta, p) \in$ $\operatorname{Dom}(H), p \in(0,1)$, we take $Z_{n}$ and $w_{n}$ as in Remark 4.1. By Lemma 3.2, we can assume that

$$
\int_{Z_{n}} \nabla w_{n} d y \rightarrow \hat{\nu} \in E(\xi, p), \quad(B-A) \hat{\nu}=\Lambda_{p} \xi-\eta .
$$

Jensen's inequality, $w_{n}$-periodic, and $\left|Z_{n}\right|=p$ give

$$
\begin{aligned}
\int_{Y} F\left(\xi+\nabla w_{n}\right) d y & =\int_{Z_{n}} F\left(\xi+\nabla w_{n}\right) d y+\int_{Y \backslash Z_{n}} F\left(\xi+\nabla w_{n}\right) d y \\
& \geq p F\left(\xi+\frac{1}{p} \int_{Z_{n}} \nabla w_{n} d y\right)+(1-p) F\left(\xi-\frac{1}{1-p} \int_{Z_{n}} \nabla w_{n} d y\right) .
\end{aligned}
$$

By taking the limit in this inequality we deduce that

$$
H(\xi, \eta, p)=\lim _{n \rightarrow \infty} \int_{Y} F\left(\xi+\nabla w_{n}\right) d y \geq p F\left(\xi+\frac{\hat{\nu}}{p}\right)+(1-p) F\left(\xi-\frac{\hat{\nu}}{1-p}\right),
$$

and then (4.14).

To prove Theorem 4.5 we need the following corrector result. We use some ideas which appear in the proof of Theorem 3.1 given in [1] and [11].

LEMMA 5.5. We consider a bounded open set $\Omega \subset \mathbf{R}^{N}$, a sequence of matrices $M_{n} \in L^{\infty}\left(\Omega ; \mathcal{M}_{N}\right)$, uniformly bounded and elliptic, and a sequence $u_{n} \in H^{1}(\Omega)$. We assume that $u_{n}$ converges weakly in $H^{1}(\Omega)$ to a function $u$ and that $-\operatorname{div} M_{n} \nabla u_{n}$ is compact in $H^{-1}(\Omega)$. For $\varepsilon>0$ small enough, we take

$$
\Omega_{\varepsilon}=\{x \in \Omega: \operatorname{dis}(x, \partial \Omega)>\varepsilon\},
$$

and, for $h \in\left(0, \frac{\varepsilon}{\sqrt{N}}\right)$, we define $w_{n}^{h} \in L^{2}\left(\Omega_{\varepsilon} ; H_{\sharp}^{1}(Y)\right)$ as the unique solution of

$$
\left\{\begin{array}{l}
w_{n}^{h}(x, .) \in H_{\sharp}^{1}(Y), \quad \int_{Y} w_{n}^{h}(x, y) d y=0, \\
-\operatorname{div}_{y} M_{n}(x+h y)\left(\nabla u(x)+\nabla_{y} w_{n}^{h}(x, y)\right)=0 \quad \text { in } \mathbf{R}^{N}, \text { a.e. } x \in \Omega_{\varepsilon} .
\end{array}\right.
$$

Then we have

$$
\lim _{h \rightarrow 0} \limsup _{n \rightarrow \infty}\left\|\nabla u_{n}(x+h y)-\nabla u-\nabla_{y} w_{n}^{h}\right\|_{L^{2}\left(\Omega_{\varepsilon} \times Y\right)^{N}}=0 .
$$

Copyright (c) by SIAM. Unauthorized reproduction of this article is prohibited. 
Proof. By extracting a subsequence if necessary, we can assume that $M_{n} \mathrm{H}$ converges to a matrix-valued function $M$. Then, by (5.17), we get

$$
\nabla u(x) \cdot y+w_{n}^{h}(x, y) \rightarrow \nabla u(x) \cdot y+w^{h}(x, y) \text { in } H^{1}(Y), \text { a.e. } x \in \Omega_{\varepsilon},
$$

when $n$ tends to infinity, with $w^{h} \in L^{2}\left(\Omega_{\varepsilon} ; H_{\sharp}^{1}(Y)\right)$ the unique solution of the problem

$$
\left\{\begin{array}{l}
w^{h}(x, .) \in H_{\sharp}^{1}(Y), \quad \int_{Y} w^{h}(x, y) d y=0, \\
-\operatorname{div}_{y}\left(M(x+h y)\left(\nabla u(x)+\nabla_{y} w^{h}(x, y)\right)\right)=0 \text { in } \mathbf{R}^{N}, \text { a.e. } x \in \Omega_{\varepsilon} .
\end{array}\right.
$$

By using in (5.20) the fact that $M(x+h y)$ converges to $M$ strongly in $L^{q}\left(\Omega_{\varepsilon} \times Y ; \mathcal{M}_{N}\right)$, $1 \leq q<+\infty$, and $*$-weakly in $L^{\infty}\left(\Omega_{\varepsilon} \times Y ; \mathcal{M}_{N}\right)$, when $h$ tends to zero, it is easy to prove that

$$
w^{h} \rightarrow 0 \text { in } L^{2}\left(\Omega_{\varepsilon} ; H_{\sharp}^{1}(Y)\right) .
$$

On the other hand, the strong convergence in $H^{-1}(\Omega)$ of $-\operatorname{div} M_{n} \nabla u_{n}$ implies that

$$
-\operatorname{div}_{y}\left[M_{n}(x+h y) \nabla u_{n}(x+h y)\right] \rightarrow-\operatorname{div}_{y}[M(x+h y) \nabla u(x+h y)] \text { in } H^{-1}(Y)
$$

for every $x \in \Omega_{\varepsilon}$, when $n$ tends to infinity. Thanks to (5.17), we can then apply the div-curl lemma (see, e.g., [23], [28]) to deduce that

$$
\begin{aligned}
& M_{n}(x+h y)\left(\nabla u_{n}(x+h y)-\nabla u-\nabla_{y} w_{n}^{h}\right) \cdot\left(\nabla u_{n}(x+h y)-\nabla u-\nabla_{y} w_{n}^{h}\right) \\
& \rightarrow M(x+h y)\left(\nabla u(x+h y)-\nabla u-\nabla_{y} w^{h}\right) \cdot\left(\nabla u(x+h y)-\nabla u-\nabla_{y} w^{h}\right)
\end{aligned}
$$

in the sense of the distributions in $Y$, for a.e. $x \in \Omega_{\varepsilon}$, when $n$ tends to infinity. By Meyer's theorem this convergence holds in $L^{1}(Y)$ weakly, for a.e. $x \in \Omega_{\varepsilon}$, and thus we have

$$
\begin{aligned}
& \int_{Y} M_{n}(x+h y)\left(\nabla u_{n}(x+h y)-\nabla u-\nabla_{y} w_{n}^{h}\right) \cdot\left(\nabla u_{n}(x+h y)-\nabla u-\nabla_{y} w_{n}^{h}\right) d y \\
& \rightarrow \int_{Y} M(x+h y)\left(\nabla u(x+h y)-\nabla u-\nabla_{y} w^{h}\right) \cdot\left(\nabla u(x+h y)-\nabla u-\nabla_{y} w^{h}\right) d y
\end{aligned}
$$

for a.e. $x \in \Omega_{\varepsilon}$. Moreover, by (5.17) there exists $C>0$ such that $\left\|\nabla u+\nabla_{y} w_{n}^{h}\right\|_{L^{2}(Y)^{N}} \leq$ $C|\nabla u|$ a.e. in $\Omega_{\varepsilon}$, and so, for another constant $C$, we have

$$
\begin{aligned}
& \int_{Y} M_{n}(x+h y)\left(\nabla u_{n}(x+h y)-\nabla u-\nabla_{y} w_{n}^{h}\right) \cdot\left(\nabla u_{n}(x+h y)-\nabla u-\nabla_{y} w_{n}^{h}\right) d y \\
& \leq \frac{C}{h^{N}} \int_{\Omega}\left|\nabla u_{n}\right|^{2} d x+C|\nabla u|^{2}, \text { a.e. in } \Omega_{\varepsilon},
\end{aligned}
$$

which together with (5.22) allows us to apply the Lebesgue dominated convergence theorem to deduce that

$$
\begin{aligned}
& \int_{\Omega_{\varepsilon}} \int_{Y} M_{n}(x+h y)\left(\nabla u_{n}(x+h y)-\nabla u-\nabla_{y} w_{n}^{h}\right) \cdot\left(\nabla u_{n}(x+h y)-\nabla u-\nabla_{y} w_{n}^{h}\right) d y d x \\
& \rightarrow \int_{\Omega_{\varepsilon}} \int_{Y} M(x+h y)\left(\nabla u(x+h y)-\nabla u-\nabla_{y} w^{h}\right) \cdot\left(\nabla u(x+h y)-\nabla u-\nabla_{y} w^{h}\right) d y d x
\end{aligned}
$$

Copyright $@$ by SIAM. Unauthorized reproduction of this article is prohibited. 
when $n$ tends to infinity, for every $h \in(0, \varepsilon / \sqrt{N})$. By (5.21), the right-hand side of this equality tends to zero when $h$ tends to zero, and then, thanks to the ellipticity of $M$, we get (5.18).

Proof of Theorem 4.5. In the proof we will separate the cases $N=1$ and $N \geq 2$. We make this distinction because for $N \geq 2$ we will use a convexity property of the set $K(A, B, p) \xi$ which does not hold for $N=1$ (see Step 3 in the proof). On the other hand, we think it is interesting to show that the one-dimensional case follows by using elementary arguments.

The proof of the theorem will be divided in three steps.

In Step 1 we will consider the case $N=1$, while steps 2 and 3 are devoted to $N \geq 2$.

In Step 2 we will prove the inequality

$$
\left\{\begin{array}{l}
\liminf _{n \rightarrow \infty} \mathcal{F}\left(u_{n}, \sigma_{n}, \theta_{n}\right) \geq \overline{\mathcal{F}}(u, \sigma, \theta) \quad \forall(u, \sigma, \theta) \in H^{1}(\Omega) \times L^{2}(\Omega)^{N} \times L^{\infty}(\Omega) \\
\forall\left(u_{n}, \sigma_{n}, \theta_{n}\right) \in H^{1}(\Omega) \times L^{2}(\Omega)^{N} \times L^{\infty}(\Omega),\left(u_{n}, \sigma_{n}, \theta_{n}\right) \stackrel{\mathcal{T}}{\rightarrow}(u, \sigma, \theta) .
\end{array}\right.
$$

The proof of (5.23) will follow from Lemma 5.5, which provides an approximation of $\nabla u_{n}$ in the strong topology of $H^{1}(\omega)(\omega \subset \subset \Omega)$ by using periodic homogenization.

In Step 3 we prove that for every $(u, \sigma, \theta) \in H^{1}(\Omega) \times L^{2}(\Omega)^{N} \times L^{\infty}(\Omega)$ there exists $\left(u_{n}, \sigma_{n}, \theta_{n}\right) \in H^{1}(\Omega) \times L^{2}(\Omega)^{N} \times L^{\infty}(\Omega)$ such that

$$
\left(u_{n}, \sigma_{n}, \theta_{n}\right) \stackrel{\mathcal{T}}{\rightarrow}(u, \sigma, \theta), \quad \limsup _{n \rightarrow \infty} \mathcal{F}\left(u_{n}, \sigma_{n}, \theta_{n}\right) \leq \overline{\mathcal{F}}(u, \sigma, \theta)
$$

which joined to (5.23) will give the proof of Theorem 4.5 in the case $N \geq 2$. The main idea to prove the existence of $\left(u_{n}, \sigma_{n}, \theta_{n}\right)$ satisfying (5.24) will be to use an approximation by finite elements of $(u, \sigma, \theta)$ which reduces the problem to the case where there exists a triangulation $\tau$ such that $\nabla u, \sigma$, and $\theta$ are constant in each element of $\tau$.

Step 1. Let us first prove the result for $N=1$.

Consider $(u, \sigma, \theta) \in H^{1}(\Omega) \times L^{2}(\Omega) \times L^{\infty}(\Omega)$ and a sequence $\left(u_{n}, \sigma_{n}, \theta_{n}\right) \in$ $H^{1}(\Omega) \times L^{2}(\Omega) \times L^{\infty}(\Omega)$ which $\mathcal{T}$-converges to $(u, \sigma, \theta)$. Let us prove that

$$
\overline{\mathcal{F}}(u, \sigma, \theta) \leq \liminf _{n \rightarrow \infty} \mathcal{F}\left(u_{n}, \sigma_{n}, \theta_{n}\right) .
$$

By definition (4.18) of $\mathcal{F}$, it is enough to consider the case where there exists a sequence of measurable sets $\omega_{n} \subset \Omega$ such that

$$
\theta_{n}=\chi_{\omega_{n}}, \sigma_{n}=\left(A \chi_{\omega_{n}}+B \chi_{\Omega \backslash \omega_{n}}\right) \frac{d u_{n}}{d x} \text { a.e. in } \Omega,
$$

and thus

$$
\frac{d u_{n}}{d x}=\frac{\sigma_{n}}{A} \chi_{\omega_{n}}+\frac{\sigma_{n}}{B} \chi_{\Omega \backslash \omega_{n}} \text { a.e. in } \Omega .
$$

Since $\sigma_{n}$ converges weakly to $\sigma$ in $L^{2}(\Omega)$ and $\frac{d \sigma_{n}}{d x}$ converges strongly to $\frac{d \sigma}{d x}$ in $H^{-1}(\Omega)$, we have that $\sigma_{n}$ converges strongly to $\sigma$ in $L^{2}(\Omega)$. So by (5.26) we get

$$
\frac{d u}{d x}=\left(\frac{\theta}{A}+\frac{1-\theta}{B}\right) \sigma \text { a.e. in } \Omega
$$

Copyright $@$ by SIAM. Unauthorized reproduction of this article is prohibited. 
and

$$
\begin{aligned}
& \lim _{n \rightarrow \infty} \mathcal{F}\left(u_{n}, \sigma_{n}, \theta_{n}\right)=\lim _{n \rightarrow \infty} \int_{\Omega} F\left(\frac{d u_{n}}{d x}\right) d x \\
& =\lim _{n \rightarrow \infty} \int_{\Omega}\left(F\left(\frac{\sigma_{n}}{A}\right) \chi_{\omega_{n}}+F\left(\frac{\sigma_{n}}{B}\right) \chi_{\Omega \backslash \omega_{n}}\right) d x=\int_{\Omega}\left(F\left(\frac{\sigma}{A}\right) \theta+F\left(\frac{\sigma}{B}\right)(1-\theta)\right) d x .
\end{aligned}
$$

By (4.9), we have then proved that $(u, \sigma, \theta)$ belongs to $\operatorname{Dom}(H)$ a.e. in $\Omega$ and

$$
\overline{\mathcal{F}}(u, \sigma, \theta)=\int_{\Omega} H\left(\frac{d u}{d x}, \sigma, \theta\right) d x=\lim _{n \rightarrow \infty} \mathcal{F}\left(u_{n}, \sigma_{n}, \theta_{n}\right) .
$$

To finish the proof of Step 1, we need to prove that for every $(u, \sigma, \theta) \in H^{1}(\Omega) \times$ $L^{2}(\Omega) \times L^{\infty}(\Omega)$ there exists $\left(u_{n}, \sigma_{n}, \theta_{n}\right) \in H^{1}(\Omega) \times L^{2}(\Omega) \times L^{\infty}(\Omega)$, which $\mathcal{T}$-converges to $(u, \sigma, \theta)$ and satisfies

$$
\limsup _{n \rightarrow \infty} \mathcal{F}\left(u_{n}, \sigma_{n}, \theta_{n}\right) \leq \overline{\mathcal{F}}(u, \sigma, \theta) .
$$

Clearly, it is enough to consider the case where $(u, \sigma, \theta) \in \operatorname{Dom}(H)$ a.e. in $\Omega$, but then $\theta \in[0,1]$ a.e. in $\Omega$, and so there exists $\omega_{n} \subset \Omega$ such that $\chi_{\omega_{n}}$ converges weakly-* in $L^{\infty}(\Omega)$ to $\theta$. By taking

$$
\theta_{n}=\chi_{\omega_{n}}, \quad \sigma_{n}=\sigma, \quad \frac{d u_{n}}{d x}=\frac{\sigma}{A} \chi_{\omega_{n}}+\frac{\sigma}{B} \chi_{\Omega \backslash \omega_{n}}
$$

and reasoning as above, we deduce (5.27).

In the remainder of the proof we always assume that $N \geq 2$.

Step 2. Let us prove (5.23).

We can assume that

$$
\liminf _{n \rightarrow \infty} \mathcal{F}\left(u_{n}, \sigma_{n}, \theta_{n}\right)<+\infty,
$$

and thus, by extracting a subsequence if necessary, there exists a sequence of measurable sets $\omega_{n} \subset \Omega$ such that $\theta_{n}=\chi_{\omega_{n}}$ and $\sigma_{n}=M_{n} \nabla u_{n}$, with $M_{n}=A \chi_{\omega_{n}}+B \chi_{\Omega \backslash \omega_{n}}$. By using the compactness theorem for the $H$-convergence (see, e.g., [1], [11], [22], [26], [27]), we can also assume that there exists an elliptic matrix $M \in L^{\infty}\left(\Omega ; \mathcal{M}_{N}^{s}\right)$ such that $M_{n} H$-converges to $M$. Since $\operatorname{div} \sigma_{n}$ converges strongly to $\operatorname{div} \sigma$ in $H^{-1}(\Omega)$, and $M_{n} \nabla u_{n}=\sigma_{n}$, we have

$$
\sigma=M \nabla u \text { a.e. in } \Omega \text {. }
$$

In order to prove (5.23), we consider $\varepsilon>0$. By defining $\Omega_{\varepsilon}$ by (5.16) and taking $h \in(0, \varepsilon / \sqrt{N})$ in such a way that $\Omega_{\varepsilon} \subset \Omega-h y$ for every $y \in Y$, we have

$$
\begin{aligned}
\int_{\Omega} F\left(\nabla u_{n}\right) d x & =\int_{\Omega-h y} F\left(\nabla u_{n}(x+h y)\right) d x \\
& =\int_{\Omega_{\varepsilon}} F\left(\nabla u_{n}(x+h y)\right) d x+\int_{(\Omega-h y) \backslash \Omega_{\varepsilon}} F\left(\nabla u_{n}(x+h y)\right) d x \\
& =\int_{\Omega_{\varepsilon}} F\left(\nabla u_{n}(x+h y)\right) d x+\int_{\Omega \backslash\left(\Omega_{\varepsilon}+h y\right)} F\left(\nabla u_{n}\right) d x
\end{aligned}
$$

Copyright $@$ by SIAM. Unauthorized reproduction of this article is prohibited. 
and so, by integrating in $y \in Y$, we have

$$
\int_{\Omega} F\left(\nabla u_{n}\right) d x=\int_{Y} \int_{\Omega_{\varepsilon}} F\left(\nabla u_{n}(x+h y)\right) d x d y+\int_{Y} \int_{\Omega \backslash\left(\Omega_{\varepsilon}+h y\right)} F\left(\nabla u_{n}\right) d x d y .
$$

By using the fact that $\Omega \backslash\left(\Omega_{\varepsilon}+h y\right) \subset \Omega \backslash \Omega_{2 \varepsilon}$ for every $y \in Y$, (4.3), and the fact that $\left|\nabla u_{n}\right|^{2}$ is equi-integrable (which follows from the definition of $\mathcal{T}$-convergence), we have

$$
\begin{aligned}
& \lim _{\varepsilon \rightarrow 0} \limsup _{n \rightarrow \infty}\left|\int_{Y} \int_{\Omega \backslash\left(\Omega_{\varepsilon}+h y\right)} F\left(\nabla u_{n}\right) d x d y\right| \\
& \leq L \lim _{\varepsilon \rightarrow 0} \limsup _{n \rightarrow \infty} \int_{\Omega \backslash \Omega_{2 \varepsilon}}\left(1+\left|\nabla u_{n}\right|\right)^{2} d x=0 .
\end{aligned}
$$

To estimate the first term of (5.30), we use the decomposition

$$
\begin{aligned}
\int_{Y} \int_{\Omega_{\varepsilon}} F\left(\nabla u_{n}(x+h y)\right) d x d y= & \int_{Y} \int_{\Omega_{\varepsilon}}\left(F\left(\nabla u_{n}(x+h y)\right)-F\left(\nabla u+\nabla_{y} w_{n}^{h}\right)\right) d x d y \\
& +\int_{\Omega_{\varepsilon}} \int_{Y} F\left(\nabla u+\nabla_{y} w_{n}^{h}\right) d y d x
\end{aligned}
$$

with $w_{n}^{h}$ given by (5.17). Thanks to (4.1) and (5.18), we have

$$
\lim _{h \rightarrow 0} \limsup _{n \rightarrow \infty} \int_{Y} \int_{\Omega_{\varepsilon}}\left|F\left(\nabla u_{n}(x+h y)\right)-F\left(\nabla u+\nabla_{y} w_{n}^{h}\right)\right| d x d y=0 \quad \forall \varepsilon>0 .
$$

To estimate the second term on the right-hand side of (5.32), we denote for a.e. $x \in \Omega_{\varepsilon}$ and a.e. $y \in Y$

$$
\begin{gathered}
Z_{n}^{h}(x)=Y \cap \frac{\omega_{n}-x}{h}, \quad \theta_{n}^{h}(x)=\left|Z_{n}^{h}(x)\right|=\int_{Y} \theta_{n}(x+h y) d y, \\
M_{n}(x+h y)=A \chi_{\omega_{n}}(x+h y)+B \chi_{\Omega \backslash \omega_{n}}(x+h y)=A \chi_{Z_{n}^{h}(x)}(y)+B \chi_{Y \backslash Z_{n}^{h}(x)}(y), \\
\sigma_{n}^{h}(x)=\int_{Y} M_{n}(x+h y)\left(\nabla u+\nabla_{y} w_{n}^{h}\right) d y .
\end{gathered}
$$

Then, by definition (5.17) of $w_{n}^{h}$ and definition (4.6) of $H$, we obtain

$$
\int_{Y} F\left(\nabla u+\nabla_{y} w_{n}^{h}\right) d y \geq H\left(\nabla u, \sigma_{n}^{h}, \theta_{n}^{h}\right), \text { a.e. in } \Omega_{\varepsilon} .
$$

By the $H$-convergence of $M_{n}$ to $M$ and the convergence in $L^{\infty}(\Omega)$ weak-* of $\theta_{n}$ to $\theta$, we have that $\sigma_{n}^{h}$ and $\theta_{n}^{h}$, respectively, converge a.e. in $\Omega_{\varepsilon}$ to $\sigma^{h} \in L^{2}\left(\Omega_{\varepsilon}\right)^{N}$ and $\theta^{h} \in L^{\infty}\left(\Omega_{\varepsilon}\right)$, defined by

$$
\sigma^{h}(x)=\int_{Y} M(x+h y)\left(\nabla u+\nabla_{y} w^{h}\right) d y, \quad \theta^{h}(x)=\int_{Y} \theta(x+h y) d y, \quad \text { a.e. } x \in \Omega_{\varepsilon} .
$$

From (4.11) and $\left\|\nabla u+\nabla_{y} w_{n}^{h}\right\|_{L^{2}(Y)} \leq C|\nabla u|$ a.e. in $\Omega$, we also have

$$
\left|H\left(\nabla u, \sigma_{n}^{h}, \theta_{n}^{h}\right)\right| \leq C(1+|\nabla u|)^{2} \quad \text { a.e. in } \Omega_{\varepsilon} .
$$

Copyright $@$ by SIAM. Unauthorized reproduction of this article is prohibited. 
Then, by (5.34), the lower semicontinuity of $H$, and Fatou's lemma we deduce that

$$
\begin{aligned}
\liminf _{n \rightarrow \infty} \int_{\Omega_{\varepsilon}} \int_{Y} F\left(\nabla u+\nabla_{y} w_{n}^{h}\right) d y d x & \geq \liminf _{n \rightarrow \infty} \int_{\Omega_{\varepsilon}} H\left(\nabla u, \sigma_{n}^{h}, \theta_{n}^{h}\right) d x \\
& \geq \int_{\Omega_{\varepsilon}} H\left(\nabla u, \sigma^{h}, \theta^{h}\right) d x
\end{aligned}
$$

for every $h \in(0, \varepsilon / \sqrt{N})$. By now using the fact that, for $h$ tending to zero, $\sigma^{h}$ converges strongly to $\sigma$ in $L^{2}\left(\Omega_{\varepsilon}\right)^{N}$ and $\theta^{h}$ converges to $\theta$ strongly in $L^{r}\left(\Omega_{\varepsilon}\right), 1 \leq r<$ $+\infty$, weak-* in $L^{\infty}\left(\Omega_{\varepsilon}\right)$, we can use again the lower semicontinuity of $H$ and Fatou's lemma to obtain

$$
\liminf _{h \rightarrow 0} \liminf _{n \rightarrow \infty} \int_{\Omega_{\varepsilon}} \int_{Y} F\left(\nabla u+\nabla_{y} w_{n}^{h}\right) d y d x \geq \int_{\Omega_{\varepsilon}} H(\nabla u, \sigma, \theta) d x .
$$

By (5.30), (5.31), (5.32), (5.33), and (5.35), by passing to the limit in (5.29), first in $n$, then in $h$, and then in $\varepsilon$, we have

$$
\liminf _{n \rightarrow \infty} \int_{\Omega} F\left(\nabla u_{n}\right) d x \geq \int_{\Omega} H(\nabla u, \sigma, \theta) d x .
$$

This proves (5.23).

Step 3. Let us prove that for every $(u, \sigma, \theta) \in H^{1}(\Omega) \times L^{2}(\Omega)^{N} \times L^{\infty}(\Omega)$ there exists $\left(u_{n}, \sigma_{n}, \theta_{n}\right) \in H^{1}(\Omega) \times L^{2}(\Omega)^{N} \times L^{\infty}(\Omega)$, which satisfies (5.24).

It is enough to consider the case where $0 \leq \theta \leq 1$ and $\sigma \in \mathcal{K}(A, B, \theta) \nabla u$ a.e. in $\Omega$.

We consider an open cube $Q$, with $\bar{\Omega} \subset Q$, and a prolongation of $u$, still denoted by $u$, in $H_{0}^{1}(Q)$. This prolongation exists thanks to $\Omega$ being smooth. We also extend $\theta$ and $\sigma$ to the whole $Q$ by $\theta=1, \sigma=A \nabla u$ a.e. in $Q \backslash \Omega$. The multiapplication $x \in \Omega \mapsto\{\tilde{M} \in \mathcal{K}(A, B, \theta(x)): \tilde{M} \nabla u(x)=\sigma(x)\}$ is closed and measurable. The measurability follows by using the fact that for every closed set $\mathcal{C} \subset \mathcal{M}_{N}^{s}$ we have

$$
\{x \in \Omega: \exists \tilde{M} \in \mathcal{C} \cap \mathcal{K}(A, B, \theta(x)) \text {, with } \tilde{M} \nabla u(x)=\sigma(x)\}=\bigcap_{m \in \mathbf{N}}(\nabla u, \sigma, \theta)^{-1}\left(K_{m}\right),
$$

where

$$
K_{m}=\left\{(\xi, \eta, p) \in \mathbf{R}^{N} \times \mathbf{R}^{N} \times[0,1]: \exists \tilde{M} \in \mathcal{K}(A, B, p) \cap \mathcal{C} \text { with }|\tilde{M} \xi-\eta| \leq \frac{1}{m}\right\}
$$

is closed for every $m \in \mathbf{N}$. Thus (see, e.g., [7]) there exists $M \in L^{\infty}\left(\Omega ; \mathcal{M}_{N}^{s}\right)$ such that $M \in \mathcal{K}(A, B, \theta)$ and $M \nabla u=\sigma$ a.e. in $Q$.

For a regular sequence of triangulations $\tau_{n}=\left\{T_{n}^{k}\right\}_{1 \leq k \leq k_{n}}$ of $Q$ by $N$-simplex, whose diameter tends to zero when $n$ tends to infinity (see, e.g., [8]), we consider the space of finite elements

$$
V_{n}=\left\{v_{n} \in C_{0}^{0}(\bar{Q}): v_{n} \text { is affine in } T_{n}^{k} \quad \forall k \in\left\{1, \ldots, k_{n}\right\}\right\} .
$$

Then we define $\hat{u}_{n}$ as the solution of

$$
\left\{\begin{array}{l}
\hat{u}_{n} \in V_{n}, \\
\int_{Q} M \nabla \hat{u}_{n} \nabla v=\int_{Q} \sigma \nabla v d x \quad \forall v \in V_{n},
\end{array}\right.
$$

Copyright (c) by SIAM. Unauthorized reproduction of this article is prohibited. 
and we take

$$
\hat{\theta}_{n}=\sum_{k=1}^{k_{n}}\left(\frac{1}{\left|T_{n}^{k}\right|} \int_{T_{n}^{k}} \theta d x\right) \chi_{T_{n}^{k}}, \quad \hat{\sigma}_{n}=\sum_{k=1}^{k_{n}}\left(\frac{1}{\left|T_{n}^{k}\right|} \int_{T_{n}^{k}} M \nabla \hat{u}_{n} d x\right) \chi_{T_{n}^{k}} .
$$

The sequence $\hat{\theta}_{n}$ converges to $\theta$ in $L^{r}(Q), 1 \leq r<+\infty$, and in $L^{\infty}(Q)$ weak-*. Since $\tau_{n}$ is regular, and $\sigma=M \nabla u$ a.e. in $Q$, we also have that $\hat{u}_{n}$ converges strongly in $H_{0}^{1}(Q)$ to $u$ (see, e.g., [8]). Thus, $M \nabla \hat{u}_{n}$ and then $\hat{\sigma}_{n}$ converge strongly to $\sigma$ in $L^{2}(Q)^{N}$. By Egorov's theorem, there exist a subsequence of $n$, still denoted by $n$, and a sequence $Q_{n}$ of closed subsets of $Q$ such that

$$
\left|Q-Q_{n}\right|<\frac{1}{n}, \quad C\left(\left|\nabla \hat{u}_{n}-\nabla u\right|+|\nabla u|\left|\hat{\theta}_{n}-\theta\right|^{\rho}\right)+\left|\hat{\sigma}_{n}-\sigma\right|<\frac{1}{n} \text { in } Q_{n},
$$

with $C>0$ and $\rho \in(0,1)$ given by Lemma 5.3.

Since $\nabla \hat{u}_{n}$ is constant in every $N$-simplex $T_{n}^{k}$ of $\tau_{n}$ and, for every $\xi \in \mathbf{R}^{N}$, the set $\mathcal{K}(A, B, p) \xi$ is closed in $\mathbf{R}^{N}$ and satisfies the following convexity property (this property does not hold for $N=1$, and so it is the reason to prove the cases $N=1$, $N \geq 2$ separately):

$\lambda \mathcal{K}\left(A, B, p_{1}\right) \xi+(1-\lambda) \mathcal{K}\left(A, B, p_{2}\right) \xi \subset \mathcal{K}\left(A, B, \lambda p_{1}+(1-\lambda) p_{2}\right) \xi \quad \forall \lambda, p_{1}, p_{2} \in[0,1]$,

we have

$$
\hat{\sigma}_{n}=\left(\frac{1}{\left|T_{n}^{k}\right|} \int_{T_{n}^{k}} M d x\right) \nabla \hat{u}_{n} \in \mathcal{K}\left(A, B, \frac{1}{\left|T_{n}^{k}\right|} \int_{T_{n}^{k}} \theta d x\right) \nabla \hat{u}_{n}
$$

in $T_{n}^{k}$, for every $k \in\left\{1, \ldots, k_{n}\right\}$, i.e., $\hat{\sigma}_{n} \in \mathcal{K}\left(A, B, \hat{\theta}_{n}\right) \nabla \hat{u}_{n}$ a.e. in $Q$, or equivalently

$$
\left(\nabla \hat{u}_{n}, \hat{\sigma}_{n}, \hat{\theta}_{n}\right) \in \operatorname{Dom}(H) \text { a.e. in } Q \text {. }
$$

We consider an $N$-simplex $T_{n}^{k}$, with $k \in\left\{1, \ldots, k_{n}\right\}$, and we denote by $\xi_{n}^{k}=\nabla \hat{u}_{n}$, $\eta_{n}^{k}=\hat{\sigma}_{n}$, and $p_{n}^{k}=\hat{\theta}_{n}$ the constant values of $\nabla \hat{u}_{n}, \hat{\sigma}_{n}$, and $\hat{\theta}_{n}$, respectively, in $T_{n}^{k}$. From definition (4.5) of $H_{\frac{2}{n}}\left(\xi_{n}^{k}, \eta_{n}^{k}, p_{n}^{k}\right)$, there exists $Z_{n}^{k} \subset Y$, with $\left|Z_{n}^{k}\right|=p_{n}^{k}$, such that, by defining $S_{n}^{k} \in L_{\sharp}^{\infty}\left(Y ; \mathcal{M}_{N}^{s}\right)$ by $S_{n}^{k}=A \chi_{Z_{n}^{k}}+B \chi_{Y \backslash Z_{n}^{k}}$ a.e. in $Y$, and taking $w_{n}^{k}$ the solution of

$$
\left\{\begin{array}{l}
w_{n}^{k} \in H_{\sharp}^{1}(Y), \quad \int_{Y} w_{n}^{k} d y=0, \\
-\operatorname{div} S_{n}^{k}\left(\xi_{n}^{k}+\nabla w_{n}^{k}\right)=0 \text { in } \mathbf{R}^{N},
\end{array}\right.
$$

we have

$$
\left|\int_{Y} S_{n}^{k}\left(\xi_{n}^{k}+\nabla w_{n}^{k}\right) d y-\eta_{n}^{k}\right|<\frac{2}{n}
$$

and

$$
\int_{Y} F\left(\xi_{n}^{k}+\nabla w_{n}^{k}\right) d y<H_{\frac{2}{n}}\left(\xi_{n}^{k}, \eta_{n}^{k}, p_{n}^{k}\right)+\frac{1}{n}
$$

We take

$$
\check{\sigma}_{n}=\sum_{k=1}^{k_{n}}\left(\int_{Y} S_{n}^{k}\left(\xi_{n}^{k}+\nabla w_{n}^{k}\right) d y\right) \chi_{T_{n}^{k}}
$$

Copyright $@$ ( ) by SIAM. Unauthorized reproduction of this article is prohibited. 
and by (5.37), we observe that

$$
\int_{Q}\left|\check{\sigma}_{n}-\hat{\sigma}_{n}\right|^{2} d x<\frac{4}{n^{2}}|Q| .
$$

For $n \in \mathbf{N}$, and $\varepsilon>0$, we define

$$
\begin{aligned}
& \omega_{n, \varepsilon}=\bigcup_{k=1}^{k_{n}}\left[T_{n}^{k} \bigcap\left(\bigcup_{l \in \mathbf{Z}^{N}}\left(\varepsilon l+\varepsilon Z_{n}^{k}\right)\right)\right], \\
& M_{n, \varepsilon}=A \chi_{\omega_{n, \varepsilon}}+B\left(1-\chi_{\omega_{n, \varepsilon}}\right),
\end{aligned}
$$

and $u_{n, \varepsilon} \in H_{0}^{1}(Q)$ as the solution of

$$
-\operatorname{div} M_{n, \varepsilon} \nabla u_{n, \varepsilon}=-\operatorname{div} \check{\sigma}_{n} \text { in } Q .
$$

Since $\left|Z_{n}^{k}\right|=p_{n}^{k}$, the sequence $\omega_{n, \varepsilon}$ satisfies

$$
\chi_{\omega_{n, \varepsilon}} \stackrel{*}{\rightarrow} \hat{\theta}_{n} \text { in } L^{\infty}(\Omega), \quad \text { when } \varepsilon \rightarrow 0 .
$$

By using the fact that $\nabla \hat{u}_{n}=\xi_{n}^{k}$ in each $T_{n}^{k}$ and the definition (5.39) of $\check{\sigma}_{n}$, we deduce by periodic homogenization (see, e.g., [1], [4], [11]) that

$$
\begin{gathered}
u_{n, \varepsilon} \rightarrow \hat{u}_{n} \text { in } H_{0}^{1}(Q), \text { when } \varepsilon \rightarrow 0, \\
M_{n, \varepsilon} \nabla u_{n, \varepsilon} \rightarrow \check{\sigma}_{n} \text { in } L^{2}(Q)^{N}, \quad \text { when } \varepsilon \rightarrow 0, \\
\nabla u_{n, \varepsilon}-\sum_{k=1}^{k_{n}}\left(\xi_{n}^{k}+\nabla w_{n}^{k}\left(\frac{x}{\varepsilon}\right)\right) \chi_{T_{n}^{k}} \rightarrow 0 \text { in } L^{2}(Q)^{N}, \text { when } \varepsilon \rightarrow 0 .
\end{gathered}
$$

By (5.45), (4.1), $\nabla w_{n}^{k}$-periodic, and (5.38), we then have

$$
\begin{aligned}
& \lim _{\varepsilon \rightarrow 0} \int_{\Omega} F\left(\nabla u_{n, \varepsilon}\right) d x=\sum_{k=1}^{k_{n}} \lim _{\varepsilon \rightarrow 0} \int_{T_{n}^{k} \cap \Omega} F\left(\xi_{n}^{k}+\nabla w_{n}^{k}\left(\frac{x}{\varepsilon}\right)\right) d x \\
& =\sum_{k=1}^{k_{n}} \int_{T_{n}^{k} \cap \Omega} \int_{Y} F\left(\xi_{n}^{k}+\nabla w_{n}^{k}\right) d y d x<\int_{\Omega} H_{\frac{2}{n}}\left(\nabla \hat{u}_{n}, \hat{\sigma}_{n}, \hat{\theta}_{n}\right) d x+\frac{|\Omega|}{n} .
\end{aligned}
$$

We consider a dense countable subset $\left\{h_{j}\right\}$ of $L^{1}(\Omega)$ and a dense countable subset $\left\{g_{j}\right\}$ of $L^{2}(\Omega)^{N}$. By using (5.42), (5.43), (5.46), for every $n \in \mathbf{N}$, we choose $\varepsilon_{n}>0$ such that

$$
\begin{gathered}
\left|\int_{\Omega}\left(\chi_{\omega_{n, \varepsilon_{n}}}-\hat{\theta}_{n}\right) h_{j} d x\right|<\frac{1}{n} \quad \forall j \in\{1, \ldots, n\}, \\
\left|\int_{\Omega}\left(M_{n, \varepsilon_{n}} \nabla u_{n, \varepsilon_{n}}-\check{\sigma}_{n}\right) g_{j} d x\right|<\frac{1}{n} \quad \forall j \in\{1, \ldots, n\}, \\
\int_{\Omega}\left|u_{n, \varepsilon_{n}}-\hat{u}_{n}\right|^{2} d x<\frac{1}{n},
\end{gathered}
$$

Copyright (c) by SIAM. Unauthorized reproduction of this article is prohibited. 


$$
\int_{\Omega} F\left(\nabla u_{n, \varepsilon_{n}}\right) d x<\int_{\Omega} H_{\frac{2}{n}}\left(\nabla \hat{u}_{n}, \hat{\sigma}_{n}, \hat{\theta}_{n}\right) d x+\frac{|\Omega|}{n} .
$$

Then we define

$$
u_{n}=u_{n, \varepsilon_{n}}, \quad \sigma_{n}=M_{n, \varepsilon_{n}} \nabla u_{n, \varepsilon_{n}}, \quad \theta_{n}=\chi_{\omega_{n, \varepsilon_{n}}} .
$$

Thanks to (5.40) and $\hat{\sigma}_{n}$ converging strongly to $\sigma$ in $L^{2}(\Omega)^{N}$, we have that $\check{\sigma}_{n}$ converges strongly to $\sigma$ in $L^{2}(\Omega)^{N}$. So, by $(5.41), u_{n}$ is bounded in $H^{1}(\Omega)$, which joined to (5.49) and $\hat{u}_{n}$ converging strongly to $u$ in $H^{1}(\Omega)$ implies that $u_{n}$ converges weakly to $u$ in $H^{1}(\Omega)$. Equation (5.41) and the strong convergence of $\check{\sigma}_{n}$ (see Remark 4.5) also give that $\left|\nabla u_{n}\right|^{2}$ is equi-integrable and that $-\operatorname{div} \sigma_{n}=-\operatorname{div} \check{\sigma}_{n}$ converges strongly to $-\operatorname{div} \sigma$ in $H^{-1}(\Omega)$. By (5.48), we also have that $\sigma_{n}$ converges weakly to $\sigma$ in $L^{2}(\Omega)^{N}$. Finally (5.47) and the convergence of $\hat{\theta}_{n}$ to $\theta$ in $L^{\infty}(Q)$ weak-* proves that $\theta_{n}$ converges to $\theta$ in $L^{\infty}(\Omega)$ weak-*. Hence, $\left(u_{n}, \sigma_{n}, \theta_{n}\right) \mathcal{T}$-converges to $(u, \sigma, \theta)$.

By (5.50) and the definition (4.18) of $\mathcal{F}$, we obtain

$$
\mathcal{F}\left(u_{n}, \sigma_{n}, \theta_{n}\right)=\int_{\Omega} F\left(\nabla u_{n}\right) d x<\int_{\Omega} H_{\frac{2}{n}}\left(\nabla \hat{u}_{n}, \hat{\sigma}_{n}, \hat{\theta}_{n}\right) d x+\frac{|\Omega|}{n} .
$$

On the other hand, by $(5.36), H_{\delta}(\xi, \eta, p)$ decreasing in $\delta$, and (5.9), we have

$$
\begin{aligned}
H_{\frac{2}{n}}\left(\nabla \hat{u}_{n}, \hat{\sigma}_{n}, \hat{\theta}_{n}\right) & \leq H_{\frac{1}{n}+\lambda_{n}(x)+\left|\hat{\sigma}_{n}-\sigma\right|}\left(\nabla \hat{u}_{n}, \hat{\sigma}_{n}, \hat{\theta}_{n}\right) \\
& \leq H_{\frac{1}{n}}(\nabla u, \sigma, \theta)+\left(1+|\nabla u|+\left|\nabla \hat{u}_{n}\right|\right)^{2-\varrho}\left(\lambda_{n}\right)^{\varrho} \\
& \leq H_{\frac{1}{n}}(\nabla u, \sigma, \theta)+\left(1+|\nabla u|+\left|\nabla \hat{u}_{n}\right|\right)^{2-\varrho} \frac{1}{n^{\varrho}},
\end{aligned}
$$

a.e. in $Q_{n}$, with

$$
\lambda_{n}=C\left(\left|\nabla\left(\hat{u}_{n}-u\right)\right|+|\nabla u|\left|\hat{\theta}_{n}-\theta\right|^{\rho}\right) .
$$

By then using (5.10), we get

$$
\begin{aligned}
& \int_{\Omega} H_{\frac{2}{n}}\left(\nabla \hat{u}_{n}, \hat{\sigma}_{n}, \hat{\theta}_{n}\right) d x \\
& \leq \int_{Q_{n} \cap \Omega} H_{\frac{1}{n}}(\nabla u, \sigma, \theta) d x+\frac{1}{n^{\varrho}} \int_{Q_{n} \cap \Omega}\left(1+|\nabla u|+\left|\nabla \hat{u}_{n}\right|\right)^{2-\varrho} d x \\
& +L \int_{\Omega \backslash Q_{n}}\left(\frac{\beta}{\alpha}\left|\nabla \hat{u}_{n}\right|\right)^{\varrho}\left(1+\frac{\beta}{\alpha}\left|\nabla \hat{u}_{n}\right|\right)^{2-\varrho} d x .
\end{aligned}
$$

By the definition of $H$ and (5.10) we can apply the Lebesgue dominated convergence theorem to deduce that

$$
H_{\frac{1}{n}}(\nabla u, \sigma, \theta) \rightarrow H(\nabla u, \sigma, \theta) \text { in } L^{1}(\Omega) .
$$

By using also the fact that $\nabla \hat{u}_{n}$ converges strongly in $L^{2}(\Omega)^{N}$ and that $\left|\Omega \backslash Q_{n}\right|$ tends to zero, we then deduce by (5.51) and (5.52) that

$$
\limsup _{n \rightarrow \infty} \mathcal{F}\left(u_{n}, \sigma_{n}, \theta_{n}\right) \leq \int_{\Omega} H(\nabla u, \sigma, \theta) d x=\overline{\mathcal{F}}(u, \sigma, \theta) .
$$

This proves (5.24).

Copyright $@$ by SIAM. Unauthorized reproduction of this article is prohibited. 
Proof of Theorem 4.3. We denote by $I$ the infimum of problem (4.4) and by $J$ the infimum of problem (4.16).

Step 1. Let us first prove that $I$ is bigger than or equal to $J$. For this purpose it is enough to observe that thanks to (4.12)

$$
\int_{\Omega} F(\nabla u) d x=\int_{\Omega} H(\nabla u, M \nabla u, \theta) d x
$$

when $\theta=\chi_{\omega}$ with $\omega \subset \Omega$ measurable and $M=A \chi_{\omega}+B \chi_{\Omega \backslash \omega}$. So in (4.4) we are minimizing the same functional as that in (4.16) but in a smaller set. This proves $I \geq J$.

Step 2. Let us now use the direct method of the calculus of variations to prove that problem (4.16) has a minimum. We consider $\theta_{n} \in L^{\infty}(\Omega)$, with $0 \leq \theta_{n} \leq 1$ a.e. in $\Omega$,

$$
\int_{\Omega} \theta_{n} d x \leq \kappa|\Omega|
$$

and $M_{n} \in \mathcal{K}(A, B, \theta)$ a.e. in $\Omega$ such that the solution $u_{n}$ of

$$
\left\{\begin{array}{l}
-\operatorname{div} M_{n} \nabla u_{n}=f \text { in } \Omega, \\
u_{n} \in H_{0}^{1}(\Omega)
\end{array}\right.
$$

satisfies

$$
\exists \lim _{n \rightarrow \infty}\left(\int_{\Omega} H\left(\nabla u_{n}, M_{n} \nabla u_{n}, \theta_{n}\right) d x+G\left(u_{n}\right)\right)=J .
$$

Thanks to $\theta_{n}$ being bounded in $L^{\infty}(\Omega)$ and the compactness of the $H$-convergence, by extracting a subsequence if necessary, we can assume that there exist $\theta \in L^{\infty}(\Omega)$ and $M \in L^{\infty}\left(\Omega ; \mathcal{M}_{N}^{s}\right)$ such that $\theta_{n}$ converges weak-* in $L^{\infty}(\Omega)$ to $\theta$ and $M_{n} H$-converges to $M$. Therefore, $\left(u_{n}, M_{n} \nabla u_{n}, \theta_{n}\right) \mathcal{T}$-converges to $(u, M \nabla u, \theta)$ (the equi-integrability of $\left|\nabla u_{n}\right|^{2}$ is an easy consequence of Meyer's theorem [19]), where

$$
\begin{gathered}
0 \leq \theta \leq 1 \text { a.e. in } \Omega, \quad \int_{\Omega} \theta d x \leq \kappa|\Omega|, \quad M \in \mathcal{K}(A, B, \theta) \text { a.e. in } \Omega, \\
\qquad \begin{array}{l}
-\operatorname{div} M \nabla u=f \text { in } \Omega, \\
u \in H_{0}^{1}(\Omega) .
\end{array}
\end{gathered}
$$

Since $\overline{\mathcal{F}}$ is lower semicontinuous for the $\mathcal{T}$-convergence and $G$ is sequentially continuous for the weak convergence in $H_{0}^{1}(\Omega)$, we have

$$
J=\lim _{n \rightarrow \infty}\left(\int_{\Omega} H\left(\nabla u_{n}, M_{n} \nabla u_{n}, \theta_{n}\right) d x+G\left(u_{n}\right)\right) \geq \int_{\Omega} H(\nabla u, M \nabla u, \theta) d x+G(u) .
$$

Thus $u, M, \theta$ is a solution of (4.16), and hence $J$ is a minimum.

Step 3. To finish the proof of Theorem 4.3, let us now prove that, for every solution $u, M, \theta$ of (4.16), there exists a sequence $\omega_{n}$ of measurable subsets of $\Omega$, with $\left|\omega_{n}\right| \leq \kappa|\Omega|$ such that the solution $u_{n}$ of

$$
\left\{\begin{array}{l}
-\operatorname{div}\left(A \chi_{\omega_{n}}+B \chi_{\Omega \backslash \omega_{n}}\right) \nabla u_{n}=f \text { in } \Omega \\
u_{n} \in H_{0}^{1}(\Omega)
\end{array}\right.
$$

Copyright (c) by SIAM. Unauthorized reproduction of this article is prohibited. 
satisfies

$$
\lim _{n \rightarrow \infty}\left(\int_{\Omega} F\left(\nabla u_{n}\right) d x+G\left(u_{n}\right)\right)=J
$$

and it is such that $\left(u_{n},\left(A \chi_{\omega_{n}}+B \chi_{\Omega \backslash \omega_{n}}\right) \nabla u_{n}, \chi_{\omega_{n}}\right) \mathcal{T}$-converges to $(u, M \nabla u, \theta)$.

By Theorem 4.5, we know that there exist $\tilde{\omega}_{n} \subset \Omega$ measurable and $\tilde{u}_{n} \in H_{0}^{1}(\Omega)$ such that, for $\tilde{M}_{n}=\left(A \chi_{\tilde{\omega}_{n}}+B \chi_{\Omega \backslash \tilde{\omega}_{n}}\right)$, the sequence $\left(\tilde{u}_{n}, \tilde{M}_{n} \nabla \tilde{u}_{n}, \chi_{\tilde{\omega}_{n}}\right) \mathcal{T}$-converges to $(u, M \nabla u, \theta)$ and

$$
\lim _{n \rightarrow \infty} \int_{\Omega} F\left(\nabla \tilde{u}_{n}\right) d x=\int_{\Omega} H(\nabla u, M \nabla u, \theta) d x .
$$

From the compactness of the $H$-convergence, we can also assume that there exists $\hat{M}$ such that $\tilde{M}_{n} H$-converges to $\hat{M}$. Then $\hat{M} \in \mathcal{K}(A, B, \theta)$ a.e. in $\Omega$ and, thanks to the definition of $\mathcal{T}$-convergence, $\hat{M} \nabla u=M \nabla u$ a.e. in $\Omega$. The weak-* convergence of $\chi_{\tilde{\omega}_{n}}$ to $\theta$ also implies that

$$
\lim _{n \rightarrow \infty}\left|\tilde{\omega}_{n}\right|=\int_{\Omega} \theta d x \leq \kappa|\Omega|
$$

Now, for every $n \in \mathbf{N}$, we consider $\omega_{n} \subset \tilde{\omega}_{n}$ measurable such that

$$
\left|\tilde{\omega}_{n} \backslash \omega_{n}\right|=\max \left\{\left|\tilde{\omega}_{n}\right|-\kappa|\Omega|, 0\right\} \rightarrow 0,
$$

and we define $u_{n}$ as the solution of (5.53).

By taking into account

$$
-\operatorname{div} \tilde{M}_{n} \nabla u_{n}=f-\operatorname{div}\left(\tilde{M}_{n}-M_{n}\right) \nabla u_{n} \text { in } \Omega,
$$

with $M_{n}=A \chi_{\omega_{n}}+B \chi_{\Omega \backslash \omega_{n}}$, the equi-integrability of $\left|\nabla u_{n}\right|^{2}$, and the fact that

$$
\lim _{n \rightarrow \infty}\left|\left\{x \in \Omega: \tilde{M}_{n}(x)-M_{n}(x) \neq 0\right\}\right|=\lim _{n \rightarrow \infty}\left|\tilde{\omega}_{n} \backslash \omega_{n}\right|=0,
$$

we have that $\left(\tilde{M}_{n}-M_{n}\right) \nabla u_{n}$ tends to zero in $L^{2}(\Omega)^{N}$ strongly. Therefore $-\operatorname{div} \tilde{M}_{n} \nabla u_{n}$ tends to $f$ strongly in $H^{-1}(\Omega)$. By the definition of $\mathcal{T}$-convergence and $-\operatorname{div} M \nabla u=f$ in $\Omega$, we also have that $-\operatorname{div} \tilde{M}_{n} \nabla \tilde{u}_{n}$ converges strongly to $f$ in $H^{-1}(\Omega)$. The divcurl lemma then gives that $\tilde{M}_{n} \nabla\left(u_{n}-\tilde{u}_{n}\right) \cdot \nabla\left(u_{n}-\tilde{u}_{n}\right)$ converges to zero in the sense of the distributions. The equi-integrability of $\left|\nabla u_{n}\right|^{2}$ and $\left|\nabla \tilde{u}_{n}\right|^{2}$ implies that this convergence holds in fact in $L^{1}(\Omega)$ weakly, and so

$$
\lim _{n \rightarrow \infty} \int_{\Omega} \tilde{M}_{n} \nabla\left(u_{n}-\tilde{u}_{n}\right) \cdot \nabla\left(u_{n}-\tilde{u}_{n}\right) d x=0
$$

which by the ellipticity of $\tilde{M}_{n}$ implies that $\nabla\left(u_{n}-\tilde{u}_{n}\right)$ converges strongly to zero in $L^{2}(\Omega)^{N}$. Thus, $\left(u_{n}, M_{n} \nabla u_{n}, \chi_{\omega_{n}}\right) \mathcal{T}$-converges to $(u, M \nabla u, \theta)$, and, by (4.1), the sequential continuity of $G$ with respect to the weak topology in $H_{0}^{1}(\Omega)$, and (5.55), we have

$$
\begin{aligned}
\lim _{n \rightarrow \infty}\left(\int_{\Omega} F\left(\nabla u_{n}\right) d x+G\left(u_{n}\right)\right) & =\lim _{n \rightarrow \infty}\left(\int_{\Omega} F\left(\nabla \tilde{u}_{n}\right) d x+G\left(u_{n}\right)\right) \\
& =\int_{\Omega} H(\nabla u, M \nabla u, \theta) d x+G(u)=J .
\end{aligned}
$$

Copyright $@$ by SIAM. Unauthorized reproduction of this article is prohibited. 
Proof of Proposition 4.6. We consider $\lambda, p_{1}, p_{2} \in[0,1], \xi_{1}, \xi_{2}, \eta_{1}, \eta_{2} \in \mathbf{R}^{N}$ such that $\eta_{1}=\eta_{2}$ for $N=1$, and $\left(\xi_{1}-\xi_{2}\right) \cdot\left(\eta_{1}-\eta_{2}\right)=0$ for $N>1$.

We take $\chi=\sum_{k \in \mathbf{Z}} \chi_{(k, k+\lambda)} \in L_{\sharp}^{\infty}(0,1)$.

If $\xi_{1} \neq \xi_{2}$, we define $u_{n} \in W_{l o c}^{1, \infty}\left(\mathbf{R}^{N}\right), \sigma_{n} \in L^{\infty}\left(\mathbf{R}^{N}\right)^{N}$, and $\theta_{n} \in L^{\infty}\left(\mathbf{R}^{N}\right)$ by

$$
\begin{aligned}
& u_{n}(x)=\xi_{2} \cdot x+\frac{1}{n} \int_{0}^{n\left(\xi_{1}-\xi_{2}\right) \cdot x} \chi(s) d s, \quad \sigma_{n}(x)=\eta_{2}+\left(\eta_{1}-\eta_{2}\right) \chi\left(n\left(\xi_{1}-\xi_{2}\right) \cdot x\right), \\
& \theta_{n}(x)=p_{2}+\left(p_{1}-p_{2}\right) \chi\left(n\left(\xi_{1}-\xi_{2}\right) \cdot x\right), \quad \text { a.e. } x \in \mathbf{R}^{N},
\end{aligned}
$$

respectively, and we observe that, by defining

$$
\begin{aligned}
& u(x)=\left(\lambda \xi_{1}+(1-\lambda) \xi_{2}\right) \cdot x, \quad \sigma(x)=\lambda \eta_{1}+(1-\lambda) \eta_{2}, \\
& \theta(x)=\lambda p_{1}+(1-\lambda) p_{2}, \quad \text { a.e. } x \in \mathbf{R}^{N},
\end{aligned}
$$

we have that, for every smooth bounded open set $\omega \subset \mathbf{R}^{N}, u_{n}$ converges to $u$ in $W^{1, \infty}(\omega)$ weak-*, $\sigma_{n}$ converges to $\sigma$ in $L^{\infty}(\omega)^{N}$ weak- $*$, and $\theta_{n}$ converges to $\theta$ in $L^{\infty}(\omega)$ weak-* and $\operatorname{div} \sigma_{n}=0$ in $\omega$. In particular, $\left(u_{n}, \sigma_{n}, \theta_{n}\right) \mathcal{T}$-converges to $(u, \sigma, \theta)$. Since the functional $\overline{\mathcal{F}}$ defined by (4.19) is lower semicontinuous in $\omega$ for the $\mathcal{T}$ convergence (use Theorem 4.5 , with $\Omega$ replaced by $\omega$ ), we get

$$
\begin{aligned}
& \int_{\omega} H\left(\lambda \xi_{1}+(1-\lambda) \xi_{2}, \lambda \eta_{1}+(1-\lambda) \eta_{2}, \lambda p_{1}+(1-\lambda) p_{2}\right) d x \\
& =\overline{\mathcal{F}}(u, \sigma, \theta) \leq \lim _{n \rightarrow \infty} \overline{\mathcal{F}}\left(u_{n}, \sigma_{n}, \theta_{n}\right) \\
& =\lim _{n \rightarrow \infty} \int_{\omega}\left(H\left(\xi_{1}, \eta_{1}, p_{1}\right) \chi\left(n\left(\xi_{1}-\xi_{2}\right) \cdot x\right)+H\left(\xi_{2}, \eta_{2}, p_{2}\right)\left(1-\chi\left(n\left(\xi_{1}-\xi_{2}\right) \cdot x\right)\right)\right) d x \\
& =\int_{\omega}\left(\lambda H\left(\xi_{1}, \eta_{1}, p_{1}\right)+(1-\lambda) H\left(\xi_{2}, \eta_{2}, p_{2}\right)\right) d x,
\end{aligned}
$$

which by the arbitrariness of $\omega$ implies (4.22) (or (4.21) if $N=1$ ).

If $\xi_{1}=\xi_{2}$ (and then $N>1$ ), we reason analogously, by taking $\zeta \neq 0$ orthogonal to $\eta_{1}-\eta_{2}$ and defining

$$
\begin{aligned}
& u_{n}(x)=\xi_{1} \cdot x, \sigma_{n}(x)=\eta_{2}+\left(\eta_{1}-\eta_{2}\right) \chi(n \zeta \cdot x), \\
& \theta_{n}(x)=p_{2}+\left(p_{1}-p_{2}\right) \chi(n \zeta \cdot x), \text { a.e. } x \in \mathbf{R}^{N} .
\end{aligned}
$$

Proof of Proposition 4.7. We recall that convex and bounded functions are locally Lipschitz in the interior of their domain. This can be easily generalized to functions of several variables, convex in each one of these variables. Then Proposition 4.6 and (4.11) prove that $H$ is continuous in the interior of its domain. On the other hand, we consider $(\xi, \eta, p) \in \operatorname{Dom}(H), p \in(0,1)$. By reasoning similarly as in the proof of Theorem 4.2, it is not difficult to show that if $p$ is close to 0 or 1 , then $H(\xi, \eta, p)$ is close to $F(\xi)$ and that if

$$
\xi \cdot\left(\Lambda_{p} \xi-\eta\right)-\left((A-B)\left(\frac{A}{p}+\frac{B}{1-p}\right)^{-1}(A-B)\right)^{\dagger}\left(\Lambda_{p} \xi-\eta\right) \cdot\left(\Lambda_{p} \xi-\eta\right)
$$

is small, then

$$
\left|H(\xi, \eta, p)-p F\left(\xi+\Xi\left(\frac{\Lambda_{p} \xi-\eta}{p}\right)\right)-(1-p) F\left(\xi-\Xi\left(\frac{\Lambda_{p} \xi-\eta}{1-p}\right)\right)\right|
$$

is small. This proves the continuity of $H$ in the boundary of its domain. 
6. Example. In this section we give an example in dimension $N \geq 2$ of a quadratic function $F$ for which we obtain an explicit expression of the function $H$ defined by (4.6) in its whole domain. This is given by the following theorem.

Theorem 6.1. We consider $N \geq 2, A, B \in \mathcal{M}_{N}^{s}$ definite positive, $s \in \mathbf{R}$ such that $s(A-B)$ is definite nonnegative, and

$$
F(\xi)=s A \xi \cdot \xi \quad \forall \xi \in \mathbf{R}^{N} .
$$

Then, for every $(\xi, \eta, p) \in \mathbf{R}^{N} \times \mathbf{R}^{N} \times(0,1)$ such that $\eta \in \mathcal{K}(A, B, p) \xi$, we have that the function $H$ defined by (4.6) is given by

$$
H(\xi, \eta, p)=s A \xi \cdot \xi+s\left(\Lambda_{p} \xi-\eta\right) \cdot \xi+\frac{s}{1-p}(A-B) \nu \cdot \nu,
$$

where $\nu$ is any vector in $\mathbf{R}^{N}$ which satisfies $(B-A) \nu=\Lambda_{p} \xi-\eta$.

In the cases $p=0, p=1$, we have

$$
H(\xi, A \xi, 1)=H(\xi, B \xi, 0)=s A \xi \cdot \xi \quad \forall \xi \in \mathbf{R}^{N} .
$$

Remark 6.1. Since $\operatorname{Ran}(A-B)=\operatorname{Ker}(A-B)^{\perp}$, it is clear that the second member of (6.1) does not depend on the choice of $\nu$. By taking $\nu=(A-B)^{\dagger}\left(\Lambda_{p} \xi-\eta\right)$, we have

$$
H(\xi, \eta, p)=s A \xi \cdot \xi+s\left(\Lambda_{p} \xi-\eta\right) \cdot \xi+\frac{s}{1-p}(A-B)^{\dagger}\left(\Lambda_{p} \xi-\eta\right) \cdot\left(\Lambda_{p} \xi-\eta\right)
$$

for every $(\xi, \eta, p) \in \mathbf{R}^{N} \times \mathbf{R}^{N} \times(0,1)$ such that $\eta \in \mathcal{K}(A, B, p) \xi$.

Proof of Theorem 6.1. By (4.12) it is enough to prove (6.1).

For $(\xi, \eta, p) \in \mathbf{R}^{N} \times \mathbf{R}^{N} \times(0,1)$ such that $\eta \in \mathcal{K}(A, B, p) \xi$, we consider $Z_{n}, S_{n}$, $w_{n}, \eta_{n}$ as in Remark 4.1, and we define

$$
\nu_{n}=\int_{Z_{n}} \nabla w_{n} d y=-\int_{Y \backslash Z_{n}} \nabla w_{n} d y .
$$

By using that $\nabla w_{n}$ has mean value zero and (3.4), we have

$$
\begin{aligned}
& \int_{Y} s A\left(\xi+\nabla w_{n}\right) \cdot\left(\xi+\nabla w_{n}\right) d y=s A \xi \cdot \xi+\int_{Y} s A \nabla w_{n} \cdot \nabla w_{n} d y \\
& =s A \xi \cdot \xi+s\left(\Lambda_{p} \xi-\eta_{n}\right) \cdot \xi+\int_{Y \backslash Z_{n}} s(A-B) \nabla w_{n} \cdot \nabla w_{n} d y .
\end{aligned}
$$

Thanks to $s(A-B)$ nonnegative, the Cauchy-Schwarz inequality proves that

$$
\begin{aligned}
& s(A-B) \nu_{n} \cdot \nu_{n}=-\int_{Y \backslash Z_{n}} s(A-B) \nabla w_{n} \cdot \nu_{n} d y \\
& \leq \int_{Y \backslash Z_{n}}\left(s(A-B) \nabla w_{n} \cdot \nabla w_{n}\right)^{\frac{1}{2}}\left(s(A-B) \nu_{n} \cdot \nu_{n}\right)^{\frac{1}{2}} d y \\
& \leq\left(\int_{Y \backslash Z_{n}} s(A-B) \nabla w_{n} \cdot \nabla w_{n} d y\right)^{\frac{1}{2}}\left((1-p) s(A-B) \nu_{n} \cdot \nu_{n}\right)^{\frac{1}{2}}
\end{aligned}
$$

and so

$$
\frac{s}{1-p}(A-B) \nu_{n} \cdot \nu_{n} \leq \int_{Y \backslash Z_{n}} s(A-B) \nabla w_{n} \cdot \nabla w_{n} d y
$$

Copyright $@$ by SIAM. Unauthorized reproduction of this article is prohibited. 
which substituted in (6.2) and then passed to the limit in $n$ proves that

$$
H(\xi, \eta, p) \geq s A \xi \cdot \xi+s\left(\Lambda_{p} \xi-\eta\right) \cdot \xi+\frac{s}{1-p}(A-B) \nu \cdot \nu,
$$

where $\nu$ equals the limit of $\nu_{n}$ (it exists for a subsequence) and satisfies $(A-B) \nu=$ $\eta-\Lambda_{p} \xi$.

In order to prove the contrary inequality, we remark that if $\eta \in \Lambda_{p} \xi+\operatorname{Ran}(A-B)$ is such that

$$
\left((A-B)\left(\frac{A}{p}+\frac{B}{1-p}\right)^{-1}(A-B)\right)^{\dagger}\left(\Lambda_{p} \xi-\eta\right) \cdot\left(\Lambda_{p} \xi-\eta\right)=\xi \cdot\left(\Lambda_{p} \xi-\eta\right),
$$

then (6.1) follows from Theorem 4.2 by taking $\nu=\Xi\left(\Lambda_{p} \xi-\eta\right)$ and using

$$
\left(\frac{A}{p}+\frac{B}{1-p}\right) \nu \cdot \nu=\left(\Lambda_{p} \xi-\eta\right) \cdot \xi
$$

Thus, it is enough to consider the case where $\eta \in \Lambda_{p} \xi+\operatorname{Ran}(A-B)$ satisfies

$$
\left((A-B)\left(\frac{A}{p}+\frac{B}{1-p}\right)^{-1}(A-B)\right)^{\dagger}\left(\Lambda_{p} \xi-\eta\right) \cdot\left(\Lambda_{p} \xi-\eta\right)<\xi \cdot\left(\Lambda_{p} \xi-\eta\right)
$$

By taking as above $\nu=\Xi\left(\Lambda_{p} \xi-\eta\right)$, we have

$$
\eta=\Lambda_{p} \xi+(A-B) \nu, \quad\left(\frac{A}{p}+\frac{B}{1-p}\right) \nu \cdot \nu<(B-A) \nu \cdot \xi .
$$

The last inequality implies that there exists $r \in(0, p)$ such that

$$
\left(\frac{1}{p-r}-\frac{1}{p}\right) A \nu \cdot \nu=(B-A) \nu \cdot \xi-\left(\frac{A}{p}+\frac{B}{1-p}\right) \nu \cdot \nu .
$$

For $\hat{\nu}=\nu /(1-r)$ we then have that $\hat{\eta}=\Lambda_{\hat{p}} \xi+(A-B) \hat{\nu}, \hat{p}=(p-r) /(1-r)$ are such that $\hat{\eta} \in \Lambda_{\hat{p}} \xi+\operatorname{Ran}(A-B)$ and that (6.4) holds with $\eta$ and $p$, respectively, replaced by $\hat{\eta}$ and $\hat{p}$. By taking into account

$$
p=r+(1-r) \hat{p}, \quad \eta=r A \xi+(1-r) \hat{\eta}
$$

and using the convexity property (4.22) of $H$, we then obtain

$$
\begin{aligned}
H(\xi, \eta, p) & \leq r H(\xi, A \xi, 1)+(1-r) H(\xi, \hat{\eta}, \hat{p}) \\
& =s A \xi \cdot \xi+s(A-B) \nu \cdot \xi+\frac{s}{1-p}(A-B) \nu \cdot \nu .
\end{aligned}
$$

This finishes the proof of Theorem 6.1.

Remark 6.2. By the proof of Theorem 6.1, we see that the sequence $Z_{n}$ given by Remark 4.1 must be chosen in such a way that the value of $\nabla w_{n}$ in $Y \backslash Z_{n}$ is close to a constant. For $N \geq 2,(\xi, \eta, p) \in \mathbf{R}^{N} \times \mathbf{R}^{N} \times(0,1), \eta \in \mathcal{K}(A, B, p) \xi, \eta \neq \Lambda_{p} \xi$, this can be carried on by using two laminations as follows: We take $\nu=\Xi\left(\Lambda_{p} \xi-\eta\right)$ and $r$ and $\hat{p}$ as in the proof of Theorem 6.1, and then we construct a matrix $M$ by a lamination of $A$ and $B$ in the direction of $\nu$ with respective proportions $\hat{p}$ and $1-\hat{p}$ and 
then a lamination of $A$ and $M$ in an orthogonal direction to $(M-A) \xi$ with respective proportions $r$ and $1-r$. This is the idea that we used in the proof of inequality (6.6).

As a particular case of Theorem 6.1, we have the following result.

Corollary 6.2. Assume that $N \geq 2, A=\alpha I, B=\beta I$, with $0<\alpha<\beta$, and for $s \in \mathbf{R}$ take $F(\xi)=s|\xi|^{2}$ for every $\xi \in \mathbf{R}^{N}$. Then the function $H$ defined by (4.6) satisfies

$$
\begin{array}{ll}
H(\xi, \eta, p)=s|\xi|^{2}+\frac{s}{\beta}\left(\Lambda_{p} \xi-\eta\right) \cdot \xi+\frac{s}{\beta(\beta-\alpha) p}\left|\Lambda_{p} \xi-\eta\right|^{2} & \text { if } s \geq 0, \\
H(\xi, \eta, p)=s|\xi|^{2}+\frac{s}{\alpha}\left(\Lambda_{p} \xi-\eta\right) \cdot \xi-\frac{s}{\alpha(\beta-\alpha)(1-p)}\left|\Lambda_{p} \xi-\eta\right|^{2} & \text { if } s \leq 0
\end{array}
$$

for every $\xi \in \mathbf{R}^{N}, p \in(0,1), \eta \in \mathcal{K}(A, B, p) \xi$.

Proof. It is enough to apply Theorem 6.1 where the matrices $A$ and $B$ and the constant $s$ must be replaced, respectively, by $\beta I, \alpha I$, and $s / \beta$ in the case $s \geq 0$ and by $\alpha I, \beta I$, and $s / \alpha$ in the case $s \leq 0$.

Remark 6.3. Corollary 6.2 and Theorem 4.3 give the relaxation of the problem

$$
\left\{\begin{array}{l}
\inf \left\{s \int_{\Omega}|\nabla u|^{2} d x\right\}, \\
-\operatorname{div}\left(\alpha \chi_{\omega}+\beta \chi_{\Omega \backslash \omega}\right) \nabla u=f \text { in } \Omega, \\
u \in H_{0}^{1}(\Omega), \\
\omega \subset \Omega \text { measurable, }|\omega| \leq \kappa|\Omega| .
\end{array}\right.
$$

In the case where $s>0$, this relaxation was obtained by Bellido and Pedregal in [3] (see also [15]) for $N=2$ and independently by Grabovsky in [10] for arbitrary $N$. The method used in the present paper is different from the ones used by these authors.

\section{REFERENCES}

[1] G. Allaire, Shape Optimization by the Homogenization Method, Appl. Math. Sci. 146, Springer-Verlag, New York, 2002.

[2] G. Allaire AND S. GutiÉRrez, Optimal design in small amplitude homogenization, M2AN Math. Model. Numer. Anal., 41 (2007), pp. 543-574.

[3] J. C. Bellido and P. Pedregal, Explicit quasiconvexification for some cost functionals depending on derivatives of the state in optimal designing, Discrete Contin. Dyn. Syst., 8 (2002), pp. 967-982.

[4] A. Bensoussan, J. L. Lions, and G. Papanicolau, Asymptotic Analysis for Periodic Structures, Stud. Math. Appl. 5, North-Holland, Amsterdam, 1978.

[5] J. Casado-Díaz, J. Couce-Calvo, and J. D. Martín-Gómez, Optimality conditions for nonconvex multistate control problems in the coefficients, SIAM J. Control Optim., 43 (2004), pp. 216-239.

[6] J. Casado-Díaz, J. Couce-Calvo, and J. D. Martín-Gómez, A density result for the variation of a material with respect to small inclusions, C. R. Math. Acad. Sci. Paris, 342 (2006), pp. 353-358.

[7] C. Castaing and M. Valadier, Convex Analysis and Measurable Multifunctions, Lecture Notes in Math. 580, Springer-Verlag, New York, 1977.

[8] P. G. Ciarlet, The Finite Element Method for Elliptic Problems, North-Holland, Amsterdam, 1978.

[9] G. Dal Maso and R. V. Kohn, The Local Character of G-closure, manuscript.

[10] Y. Grabovsky, Optimal design for two-phase conducting composites with weakly discontinuous objective functionals, Adv. Appl. Math., 27 (2001), pp. 683-704.

[11] V. V. Jikov, S. M. Kozlov, And O. A. Oleinik, Homogenization of Differential Operators and Integral Functionals, Springer-Verlag, Berlin, 1994. 
[12] R. Lipton, Configurations of nonlinear materials with electric fields that minimize $L^{p}$ norms, Phys. B, 338 (2003), pp. 48-53.

[13] R. Lipton, Relaxation through homogenization for optimal design problems with gradient constraint, J. Optim. Theory Appl., 114 (2002), pp. 27-53.

[14] R. Lipton, Stress constrained G closure and relaxation of structural design problems, Quart. Appl. Math., 62 (2004), pp. 295-321.

[15] R. Lipton And A. P. Velo, Optimal design of gradient fields with applications to electrostatics, in Nonlinear Partial Differential Equations and Their Applications, Collège de France Seminar, Vol. XIV, Stud. Math. Appl. 31, D. Cioranescu and J. L. Lions, eds., North-Holland, Amsterdam, 2002, pp. 509-532.

[16] K. A. Lurie, Applied Optimal Control Theory of Distributed Systems, Plenum Press, New York, 1993.

[17] K. A. Lurie And A. V. Cherkaev, Exact estimates of the conductivity of a binary mixture of isotropic materials, Proc. Roy. Soc. Edinburgh Sect. A, 104 (1986), pp. 21-38.

[18] F. Maestre and P. Pedregal, Quasiconvexification in 3-D for a variational reformulation of an optimal design problem in conductivity, Nonlinear Anal., 64 (2006), pp. 1962-1976.

[19] N. G. Meyers, An $L^{p}$-estimate for the gradient of solutions of second order elliptic divergence equations, Ann. Scuola Norm. Sup. Pisa (3), 17 (1963), pp. 189-206.

[20] F. Murat, Un contre-exemple pour le problème du contrôle dans les coefficients, C. R. Acad. Sci. Paris Sér. A-B, 273 (1971), pp. A708-A711.

[21] F. Murat, Théorèmes de non-existence pour des problèmes de contrôle dans les coefficients, C. R. Acad. Sci. Paris Sér. A, 274 (1972), pp. A395-A398.

[22] F. Murat and L. Tartar, H-convergence, in Topics in the Mathematical Modelling of Composite Materials, Progr. Nonlinear Differential Equations Appl. 31, L. Cherkaev and R. V. Kohn, eds., Birkaüser, Boston, 1998, pp. 21-43.

[23] F. Murat, Compacité par compensation, Ann. Scuola Norm. Sup. Pisa Cl. Sci. (4), 5 (1978), pp. $489-507$.

[24] F. Murat And L. Tartar, Calculus of variations and homogenization, in Topics in the Mathematical Modelling of Composite Materials, Progr. Nonlinear Differential Equations Appl. 31, L. Cherkaev and R. V. Kohn, eds., Birkaüser, Boston, 1998, pp. 139-174.

[25] P. Pedregal, Optimal design in two-dimensional conductivity for a general cost depending on the field, Arch. Ration. Mech. Anal., 182 (2006), pp. 367-385.

[26] S. Spagnolo, Sulla convergenza di soluzioni di equazioni paraboliche ed ellittiche, Ann. Scuola Norm. Sup. Pisa Cl. Sci. (3), 22 (1968), pp. 571-597.

[27] L. Tartar, Cours Peccot, Collège de France, Paris, 1977; part of this work appears in [22].

[28] L. TARTAR, Compensated compactness and applications to partial differential equations, nonlinear analysis and mechanics, in Heriot-Watt Symposium IV, Res. Notes Math. 39, R. J. Knops, ed., Pitman, San Francisco, 1979, pp. 136-212.

[29] L. TARTAR, Estimations fines de coefficients homogénéisés, in Ennio de Giorgi Colloquium (Paris, 1983), Res. Notes Math. 125, P. Kree, ed., Pitman, London, 1985, pp. 168-187.

[30] L. TARTAR, Remarks on optimal design problems, in Calculus of Variations, Homogenization and Continuum Mechanics, Adv. Math. Appl. Sci. 18, G. Buttazzo, G. Bouchitte, and P. Suquet, eds., World Scientific, Singapore, 1994, pp. 279-296.

[31] P. Velo, Optimal Design of Gradient Fields and Currents, Ph.D. thesis, WPI, Worcester, MA, 2000; part of this work appears in [15].

Copyright $@$ ( ) by SIAM. Unauthorized reproduction of this article is prohibited. 